\title{
Treatment of Reissner-Mindlin shells with kinks without the need for drilling rotation stabilization in an isogeometric framework
}

\author{
W. Dornisch*, S. Klinkel \\ Lehrstuhl für Baustatik und Baudynamik, RWTH Aachen, Mies-van-der-Rohe-Str. 1, 52074 Aachen, Germany
}

\begin{abstract}
This work presents a framework for the computation of complex geometries containing intersections of multiple patches with Reissner-Mindlin shell elements. The main objective is to provide an isogeometric finite element implementation which neither requires drilling rotation stabilization, nor user interaction to quantify the number of rotational degrees of freedom for every node. For this purpose, the following set of methods is presented. Control points with corresponding physical location are assigned to one common node for the finite element solution. A nodal basis system in every control point is defined, which ensures an exact interpolation of the director vector throughout the whole domain. A distinction criterion for the automatic quantification of rotational degrees of freedom for every node is presented. An isogeometric Reissner-Mindlin shell formulation is enhanced to handle geometries with kinks and allowing for arbitrary intersections of patches. The parametrization of adjacent patches along the interface has to be conforming. The shell formulation is derived from the continuum theory and uses a rotational update scheme for the current director vector. The nonlinear kinematic allows the computation of large deformations and large rotations. Two concepts for the description of rotations are presented. The first one uses an interpolation which is commonly used in standard Lagrange-based shell element formulations. The second scheme uses a more elaborate concept proposed by the authors in prior work, which increases the accuracy for arbitrary curved geometries. Numerical examples show the high accuracy and robustness of both concepts. The applicability of the proposed framework is demonstrated.
\end{abstract}

Keywords: Isogeometric analysis, Geometrical nonlinear Reissner-Mindlin shell, NURBS, Interpolation of rotations, Treatment of kinks, Shell not requiring drilling rotation stabilization

\section{Introduction}

Reissner-Mindlin shell elements are the prevailing discretization technique for the computation of complex surfaceoriented structures. Most common finite element analysis packages provide shell formulations based on the ReissnerMindlin shell theory. Several ways for the derivations of shell theories exist, see [1]. The well-known contributions of Simo et al. [2, 3, 4, 5] introduce a Reissner-Mindlin shell formulation, which is derived from the continuum theory. These papers form the fundament of [6] and of this contribution, which propose an isogeometric rotation-based Reissner-Mindlin shell formulation. In smooth areas the rotational state of Reissner-Mindlin shells is described by two rotations, which are aligned to the local geometry. No stiffness against rotations around the normal vector arises. All classical shell theories require $C^{1}$-continuous surfaces and thus strictly speaking do not cover geometries with

${ }^{*}$ Correspondence to: W. Dornisch, Lehrstuhl für Technische Mechanik, Technische Universität Kaiserslautern, Gottlieb-Daimler-Str., 67663 Kaiserslautern, Germany. E-mail: dornisch@rhrk.uni-kl.de

This is the peer reviewed version of the following article: Dornisch, W, and Klinkel, S (2014): Treatment of Reissner-Mindlin shells with kinks without the need for drilling rotation stabilization in an isogeometric framework. Comput. Methods Appl. Mech. Engrg. 276, 35-66, which has been published in final form at doi: 10.1016/j.cma.2014.03.017. (C2014. This manuscript version is made available under the CC-BY-NC-ND 4.0 license http://creativecommons.org/licenses/by-nc-nd/4.0/. 
kinks. Although precluded from a theoretical point of view, geometries with kinks and sharp folds are an important area of application in engineering practice. In [3] it is proposed to use two nodal rotations in smooth areas and three global rotations for nodes on kinks. Stiffness against three rotations arises merely from the intersection of surfaces, which is the case at kinks or for faceted surfaces. If stiffness against one rotation is very low or even zero, numerical problems arise. This can be prevented by a classification of nodes - located in smooth areas or on kinks. However, there is no general criterion for this distinction, which necessitates a manual classification. Alternatively, some kind of drilling rotation stabilization can be used. All kinds of shell formulations using $C^{0}$-continuous Lagrange basis functions - independent of the derivation of the shell theory - suffer from this problem. For an excellent overview of existing shell theories and details about the aforementioned numerical issues see [1].

The possibilities offered by the introduction of isogeometric analysis by Hughes et al. [7] have the potential to change this situation fundamentally. The exact description of the geometry with NURBS allows an automatic and appropriate treatment of both smooth regions and kinks. In the meantime several NURBS-based isogeometric shell formulations have been published. An isogeometric Kirchhoff-Love shell is proposed by Kiendl et al. [8]. Benson et al. [9] use a rotation-free shell [10] in regular regions and a Reissner-Mindlin shell [11] in regions with geometric discontinuities. The rotation-based Reissner-Mindlin shell formulation proposed by the authors [6] uses a new concept for the interpolation of the director vector adapted to the characteristics of NURBS surfaces. The computation of multi-patch geometries with kinks with a linearized version of this shell formulation is discussed in [12]. The work of Echter et al. [13] uses a difference vector formulation with transverse shear deformations instead of rotations as degrees of freedom. A solid-shell with two different methods to tackle locking phenomena is proposed in [14]. In addition to that, there are several isogeometric shell formulations which use other geometry descriptions, see e.g. [15] and [16].

Despite the presence of several shell formulations, until now, no parameter-free approach to treat shell models with kinks has been presented in an isogeometric framework. The works of Benson et al. [11,9] - implemented into the commercial FEA code LS-DYNA - rely on drilling rotation stabilization, which renders results dependent on drilling rotation stabilization factors. The Kirchhoff-Love shell formulation of Kiendl et al. [8] uses a penalty-like method to impede hinging behavior at $C^{0}$-continuous patch intersections, called bending strip method [17]. Its results depend on a penalty factor and in [9] it is shown, that the bending strip method needs aligned derivatives at intersections to work properly. To the authors' knowledge, currently no other isogeometric shell formulation does discuss the computation of geometries with kinks.

Based on the aforementioned the new aspects and the main contributions of this paper are the following.

(i) A nonlinear Reissner-Mindlin shell theory for large deformations and finite rotations is presented. The geometry is described with the shell mid-surface and an inextensible director vector. Thus, no thickness strains arise. Green-Lagrange strains are used as a measure for the strains and require the interpolation of the director vector, its derivatives and variations thereof. Basing on the previous publication [6] two methods for the interpolation of the current director vector and the other mentioned quantities are presented. The variation of the director vector is expressed as a function of the variation of nodal rotations.

(ii) Methods for the interpolation of the director vector and its variations in the presence of kinks are presented. The higher continuity of NURBS allows to neither hurt the orthogonality nor the continuity. Basically two sets of nodal basis systems are used. One set is defined patch wise and is able to interpolate the director vector exactly. Within the patches the director field is at least $C^{1}$-continuous, whereas it is discontinuous between patches. An additional globally valid set of nodal basis system is used to define unique rotation axes for every node present in the finite element solution.

(iii) The method to compute exact nodal basis systems presented in [6] is extended to multi-patch geometries. The nodal basis systems are computed patch by patch. A basis system for every control point is attained. If more than one control point is assigned to one finite element node - which is in general the case at patch intersections - one unique basis system for the node under consideration has to be chosen.

(iv) A unique criterion for the classification of nodes - in nodes on kinks and nodes in smooth regions - is provided. To the authors knowledge this work presents the first formulation which is able to make this classification automatically. The physical problem is computed in a way that neither user intervention, nor drilling rotation stabilization or penalty methods are required.

(v) The developed shell formulations, the method for the computation of nodal basis systems and the classification 
criterion are tested with the help of several test problems. A basic numerical example provides a threshold value for the newly established classification criterion. Benchmarks for multi-patch geometries with kinks test the linear and nonlinear behavior of the proposed shell formulations. Computations of geometries with kinks in the large rotation regime using the two proposed methods are compared to existing formulations.

\section{NURBS-based isogeometric analysis}

The presented approach uses bivariate NURBS surface patches to describe the geometry. Complex geometries consist in general of several patches; the total number of patches is referred to as $n_{p}$. The notation follows [6] and is extended to the needs of multi-patch analysis. Details about the basics of NURBS can be found in [18]. If not specified differently Greek indices range from 1 to 2 and Latin indices from 1 to 3 . The set of $n_{1}^{k} \times n_{2}^{k}$ control points

$$
\mathbf{B}_{i j}^{k}=\left[x_{i j k}, y_{i j k}, z_{i j k}, w_{i j k}\right]^{T}=\left[\begin{array}{c}
\mathbf{X}^{(i, j, k)} \\
w_{i j k}
\end{array}\right] \quad i=1, \ldots, n_{1}^{k} \quad j=1, \ldots, n_{2}^{k}
$$

together with the two open knot vectors

$$
\boldsymbol{\Xi}_{\alpha}^{k}=\left\{\xi_{1}^{\alpha}, \xi_{2}^{\alpha}, \cdots, \xi_{n_{\alpha}^{k}+p_{\alpha}^{k}+1}^{\alpha}\right\} \quad \alpha=1,2
$$

defines the patch $k$ with order $p_{1}^{k}$ and $p_{2}^{k}$ in the first and second parametric direction. The tensor product multiplication of two knot spans

$$
\left[\xi_{i}^{1}, \xi_{i+1}^{1}\right] \times\left[\xi_{j}^{2}, \xi_{j+1}^{2}\right] \quad i=p_{1}^{k}+1, \ldots, n_{1}^{k} \quad j=p_{2}^{k}+1, \ldots, n_{2}^{k}
$$

defines an element $e$ for the finite element discretization. Elements with an area of zero, which arise due to repeated values in the knot vector, are treated as regular elements. These zero-sized elements are detected on element level and consequently the computation of their element matrices is skipped. Every patch has its own control points $\mathbf{B}_{i j}^{k}$ and there is no connection of the patches in the CAD input data. Thus, control points with corresponding locations

$$
\mathbf{X}^{(i, j, k)}=\mathbf{X}^{\left(i_{1}, j_{1}, k_{1}\right)} \quad i=1, \ldots, n_{1}^{k} \quad j=1, \ldots, n_{2}^{k} \quad k=1, \ldots, n_{p}
$$

are assigned to the same node $\mathbf{X}_{I}=\mathbf{X}^{\left(i_{1}, j_{1}, k_{1}\right)}$ for the finite element analysis. Despite being merged to one finite element node, every control point retains its individual weight for the computation of the basis functions in (8). The index $I$ is specified by the surjective and non-bijective function

$$
I=f(i, j, k)
$$

The inverse relation

$$
(i, j, k)=g(I, e)
$$

is injective on every element $e$. Thus, summation over $I$ can be used, and it is assured, that the weight $w_{i j k}$ of the correct control point is used in (8). The number of specified basis functions having influence on an arbitrary element is defined by $n_{e n}^{k}=\left(p_{1}^{k}+1\right)\left(p_{2}^{k}+1\right)$. The basis functions for the description of the physical surface $\mathbf{X}$ of a specified patch $k$

$$
\mathbf{X}\left(\xi^{1}, \xi^{2}, k\right)=\sum_{I=1}^{n_{e n}^{k}} N_{I}\left(\xi^{1}, \xi^{2}, k\right) \mathbf{X}_{I}
$$

as well as for the approximation of finite element fields, are computed with

$$
N_{I}\left(\xi^{1}, \xi^{2}, k\right)=\frac{N_{i}^{p_{1}^{k}}\left(\xi^{1}\right) N_{j}^{p_{2}^{k}}\left(\xi^{2}\right) w_{i j k}}{W\left(\xi^{1}, \xi^{2}, k\right)}
$$

The denominator reads

$$
W\left(\xi^{1}, \xi^{2}, k\right)=\sum_{i=1}^{n_{1}^{k}} \sum_{j=1}^{n_{2}^{k}} N_{i}^{p_{1}^{k}}\left(\xi^{1}\right) N_{j}^{p_{2}^{k}}\left(\xi^{2}\right) w_{i j k}
$$


with the univariate basis functions of B-Splines defined by the Cox-de Boor formula

$$
\begin{aligned}
& p=0: \quad N_{i}^{0}(\xi)= \begin{cases}1 & \text { if } \xi_{i} \leq \xi \leq \xi_{i+1} \\
0 & \text { otherwise }\end{cases} \\
& p>0: \quad N_{i}^{p}(\xi)=\frac{\xi-\xi_{i}}{\xi_{i+p}-\xi_{i}} N_{i}^{p-1}(\xi)+\frac{\xi_{i+p+1}-\xi}{\xi_{i+p+1}-\xi_{i+1}} N_{i+1}^{p-1}(\xi),
\end{aligned}
$$

where the discrete values of the knots $\xi_{i}$ with $i=1, \ldots, n_{\alpha}^{k}+p_{\alpha}^{k}+1$ are taken from the appropriate knot vector $\boldsymbol{\Xi}_{\alpha}^{k}$. The derivative

$$
\frac{\partial}{\partial \xi} N_{i}^{p}=\frac{p}{\xi_{i+p}-\xi_{i}} N_{i}^{p-1}-\frac{p}{\xi_{i+p+1}-\xi_{i+1}} N_{i+1}^{p-1}
$$

of the univariate B-Spline basis functions is needed to compute the derivative of the NURBS basis functions

$$
\frac{\partial}{\partial \xi^{\alpha}} N_{I}\left(\xi^{1}, \xi^{2}, k\right)=w_{i j k} \frac{W \frac{\partial}{\partial \xi^{\alpha}}\left(N_{i}^{p_{1}^{k}} N_{j}^{p_{2}^{k}}\right)-\frac{\partial}{\partial \xi^{\alpha}}(W) N_{i}^{p_{1}^{k}} N_{j}^{p_{2}^{k}}}{W^{2}},
$$

where the dependence of $W$ and $N_{i}^{p}$ on the parameters $\xi^{\alpha}$ and the patch $k$ is not displayed, but self-evident. The number of elements per patch $n_{e l}^{k}=\left(n_{1}^{k}-p_{1}^{k}\right)\left(n_{2}^{k}-p_{2}^{k}\right)$ sums up to the total number of elements $n_{e l}=\sum_{k=1}^{n_{p}} n_{e l}^{k}$, which includes the zero-sized elements arising at repeated knots. The total number of control points $n_{n p}=\sum_{k=1}^{n_{p}} n_{n p}^{k}$ with $n_{n p}^{k}=n_{1}^{k} \times n_{2}^{k}$ contains every instance of corresponding control points.

\section{Reissner-Mindlin shell formulation}

The proposed Reissner-Mindlin shell formulation uses a rotational update of the inextensible director vector field to precisely describe large rotations. Large deformations can be treated as the strains are described by Green-Lagrange strains. The second Piola-Kirchhoff stress tensor is the complimentary measure for the stresses. The derivation bases on the direct approach proposed by Simo and Fox [2] and is given in detail in [6]. The variational formulation is pure displacement based and no measures against locking are considered.

A physical point on the domain in the reference, respective current configuration is given by

$$
\begin{aligned}
\boldsymbol{\Phi}\left(\xi^{i}\right) & =\mathbf{X}\left(\xi^{\alpha}\right)+\xi^{3} \mathbf{D}\left(\xi^{\alpha}\right) \\
\boldsymbol{\phi}\left(\xi^{i}\right) & =\mathbf{x}\left(\xi^{\alpha}\right)+\xi^{3} \mathbf{d}\left(\xi^{\alpha}\right),
\end{aligned}
$$

where $\mathbf{X}\left(\xi^{\alpha}\right)$ and $\mathbf{x}\left(\xi^{\alpha}\right)$ are the position vectors on the shell mid-surface. The thickness coordinate $\xi^{3}$ is in the range $-h / 2 \leq \xi^{3} \leq h / 2$, where $h$ is the shell thickness. The reference director vector $\mathbf{D}\left(\xi^{\alpha}\right)$ is collinear to the normal vector $\mathbf{N}$ and $\left\|\mathbf{D}\left(\xi^{\alpha}\right)\right\|=1$ is defined to hold. An orthogonal rotation with the rotation tensor $\mathbf{R} \in S O$ (3) to determine the current director vector $\mathbf{d}=\mathbf{R D}$ ensures the inextensibility of the shell.

The Green-Lagrange strains are fractionized into resulting shell strains

$$
\begin{aligned}
\varepsilon_{\alpha \beta} & =\frac{1}{2}\left(\mathbf{x}_{, \alpha} \cdot \mathbf{x}_{, \beta}-\mathbf{X}_{, \alpha} \cdot \mathbf{X}_{, \beta}\right) \\
\kappa_{\alpha \beta} & =\frac{1}{2}\left(\mathbf{x}_{, \alpha} \cdot \mathbf{d}_{, \beta}+\mathbf{x}_{, \beta} \cdot \mathbf{d}_{, \alpha}-\mathbf{X}_{, \alpha} \cdot \mathbf{D}_{, \beta}-\mathbf{X}_{, \beta} \cdot \mathbf{D}_{, \alpha}\right) \\
\gamma_{\alpha} & =\mathbf{x}_{, \alpha} \cdot \mathbf{d}-\mathbf{X}_{, \alpha} \cdot \mathbf{D}
\end{aligned}
$$

neglecting terms containing squares of $\xi^{3}$. The subscript comma denotes a derivation with respect to the parametric coordinates $\xi^{\alpha}$. The membrane strains $\varepsilon_{\alpha \beta}$, the curvatures $\kappa_{\alpha \beta}$ and the shear strains $\gamma_{\alpha}$ are arranged to the vector

$$
\varepsilon=\left[\varepsilon_{11}, \varepsilon_{22}, 2 \varepsilon_{12}, \kappa_{11}, \kappa_{22}, 2 \kappa_{12}, \gamma_{1}, \gamma_{2}\right]^{T}
$$


in Voigt notation. Accordingly the work-conjugate stress resultants

$$
\boldsymbol{\sigma}=\left[n^{11}, n^{22}, n^{12}, m^{11}, m^{22}, m^{12}, q^{1}, q^{2}\right]^{T}
$$

of the second Piola-Kirchhoff stress tensor are arranged listing membrane forces, bending moments and shear forces.

The weak form of the equilibrium

$$
G(\mathbf{v}, \delta \mathbf{v})=\int_{\Omega_{0}} \delta \boldsymbol{\varepsilon}^{T} \boldsymbol{\sigma} \mathrm{d} A-\int_{\Omega_{0}} \delta \mathbf{v}^{T} \overline{\mathbf{p}} \mathrm{d} A-\int_{\Gamma_{t}} \delta \mathbf{v}^{T} \overline{\mathbf{t}} \mathrm{d} s=0
$$

accounts for static Neumann conditions $\overline{\mathbf{t}}$ on the boundary $\Gamma_{t}$ and conservative surface loads $\overline{\mathbf{p}}$ on the reference midsurface. The independent displacement vector $\mathbf{v}=[\mathbf{u}, \omega]^{T}$ consists of displacements $\mathbf{u}$ and rotational parameters $\omega$. The variation of the shell strains $\delta \boldsymbol{\varepsilon}$ arising in (17) results in the components

$$
\begin{aligned}
\delta \varepsilon_{\alpha \beta} & =\frac{1}{2}\left(\delta \mathbf{x}_{, \alpha} \cdot \mathbf{x}_{, \beta}+\delta \mathbf{x}_{, \beta} \cdot \mathbf{x}_{, \alpha}\right) \\
\delta \kappa_{\alpha \beta} & =\frac{1}{2}\left(\delta \mathbf{x}_{, \alpha} \cdot \mathbf{d}_{\beta}+\delta \mathbf{x}_{, \beta} \cdot \mathbf{d}_{, \alpha}+\delta \mathbf{d}_{, \alpha} \cdot \mathbf{x}_{\beta}+\delta \mathbf{d}_{\beta} \cdot \mathbf{x}_{, \alpha}\right) \\
\delta \gamma_{\alpha} & =\delta \mathbf{x}_{, \alpha} \cdot \mathbf{d}+\delta \mathbf{d} \cdot \mathbf{x}_{, \alpha},
\end{aligned}
$$

which are arranged akin to (15). The solution of (17) with the Newton-Raphson scheme requires the linearization

$$
\mathrm{L}[G(\mathbf{v}, \delta \mathbf{v})]=G+\mathrm{D} G \cdot \Delta \mathbf{v}
$$

with

$$
\mathrm{D} G(\mathbf{v}, \delta \mathbf{v}) \cdot \Delta \mathbf{v}=\int_{\mathcal{B}_{0}} \delta \varepsilon^{T} \mathbb{C} \Delta \varepsilon+\Delta \delta \varepsilon^{T} \sigma \mathrm{d} V
$$

The matrix

$$
\mathbb{C}=\frac{\partial \sigma}{\partial \boldsymbol{\varepsilon}}
$$

depends on the chosen material law. The linearized shell strains $\Delta \varepsilon$ are obtained by replacing the operator $\delta$ with $\Delta$ in (18). The second variation $\Delta \delta \boldsymbol{\varepsilon}$ has the components

$$
\begin{aligned}
\Delta \delta \varepsilon_{\alpha \beta}= & \frac{1}{2}\left(\delta \mathbf{x}_{, \alpha} \cdot \Delta \mathbf{x}_{, \beta}+\delta \mathbf{x}_{, \beta} \cdot \Delta \mathbf{x}_{, \alpha}\right) \\
\Delta \delta \kappa_{\alpha \beta}= & \frac{1}{2}\left(\delta \mathbf{x}_{, \alpha} \cdot \Delta \mathbf{d}_{\beta}+\delta \mathbf{x}_{, \beta} \cdot \Delta \mathbf{d}_{, \alpha}+\delta \mathbf{d}_{, \alpha} \cdot \Delta \mathbf{x}_{, \beta}+\delta \mathbf{d}_{\beta} \cdot \Delta \mathbf{x}_{, \alpha}\right. \\
& \left.\quad+\mathbf{x}_{, \alpha} \cdot \Delta \delta \mathbf{d}_{\beta}+\mathbf{x}_{, \beta} \cdot \Delta \delta \mathbf{d}_{, \alpha}\right) \\
\Delta \delta \gamma_{\alpha}= & \delta \mathbf{x}_{, \alpha} \cdot \Delta \mathbf{d}+\delta \mathbf{d} \cdot \Delta \mathbf{x}_{, \alpha}+\mathbf{x}_{, \alpha} \cdot \Delta \delta \mathbf{d}
\end{aligned}
$$

\section{Isogeometric discretization}

The main focus of this contribution is the treatment of geometries with kinks. This affects only the interpolation of the director vectors $\mathbf{d}^{h}$ and the variations $\delta \mathbf{d}^{h}$ and $\Delta \delta \mathbf{d}^{h}$ thereof, which are examined in Sec. 7 in detail. The discretization of all other quantities is not affected and in exact accordance to the shell formulation presented in [6]. Thus, the general discretization is only briefly sketched in the following. For all details including the linearization of the weak form we refer to [6].

The shell middle surface

$$
\mathbf{X}=\sum_{I=1}^{n_{e n}} N_{I} \mathbf{X}_{I} \quad \mathbf{x}^{h}=\sum_{I=1}^{n_{e n}} N_{I} \mathbf{x}_{I}
$$

is interpolated with the NURBS basis functions $N_{I}$ defined in (8). Approximations are denoted by an superscript $h$ in the following. The derivatives of the NURBS basis functions defined in (12) are with respect to the parametric 
coordinates $\xi^{\alpha}$. The presented shell formulation uses local Cartesian basis systems $\mathbf{t}_{i}$ in the integration points to simplify the implementation of constitutive models. The Jacobian

$$
\mathbf{J}=\left[\begin{array}{ll}
\mathbf{X}_{, \xi^{1}} \cdot \mathbf{t}_{1} & \mathbf{X}_{, \xi^{1}} \cdot \mathbf{t}_{2} \\
\mathbf{X}_{, \xi^{2}} \cdot \mathbf{t}_{1} & \mathbf{X}_{, \xi^{2}} \cdot \mathbf{t}_{2}
\end{array}\right]
$$

transforms the derivatives

$$
\left[\begin{array}{c}
N_{I, 1} \\
N_{I, 2}
\end{array}\right]=\mathbf{J}^{-1}\left[\begin{array}{c}
N_{I, \xi^{1}} \\
N_{I, \xi^{2}}
\end{array}\right]
$$

from convective basis systems to local Cartesian basis systems. The derivatives

$$
\mathbf{X}_{, \alpha}=\sum_{I=1}^{n_{e n}} N_{I, \alpha} \mathbf{X}_{I} \quad \mathbf{x}_{, \alpha}^{h}=\sum_{I=1}^{n_{e n}} N_{I, \alpha} \mathbf{x}_{I}
$$

of the shell middle surface are thus with respect to the local Cartesian basis systems. The interpolated Green-Lagrange strains $\boldsymbol{\varepsilon}^{h}$ are attained by inserting the interpolated current director vector $\mathbf{d}^{h}$ and $\mathbf{x}^{h}$ in (14). Akin the variation of the Green-Lagrange strains $\delta \boldsymbol{\varepsilon}^{h}$ arises by inserting the variation of the current location vector

$$
\delta \mathbf{x}^{h}=\sum_{I=1}^{n_{e n}} N_{I} \delta \mathbf{u}_{I} \quad \delta \mathbf{x}_{, \alpha}^{h}=\sum_{I=1}^{n_{e n}} N_{I, \alpha} \delta \mathbf{u}_{I}
$$

and the variated current director vector $\delta \mathbf{d}^{h}$ in (18). The $\mathbf{B}_{I}$ matrix

$$
\mathbf{B}_{I}=\left[\begin{array}{cc}
N_{I, 1} \mathbf{x}_{, 1}^{h T} & \mathbf{0} \\
N_{I, 2} \mathbf{x}_{, 2}^{h T} & \mathbf{0} \\
N_{I, 1} \mathbf{x}_{, 2}^{h T}+N_{I, 2} \mathbf{x}_{, 1}^{h T} & \mathbf{0} \\
N_{I, 1} \mathbf{d}_{, 1}^{h T} & \hat{\mathbf{b}}_{11 T}^{h T} \\
N_{I, 2} \mathbf{d}_{, 2}^{h T} & \hat{\mathbf{b}}_{22 I}^{h T} \\
N_{I, 2} \mathbf{d}_{, 1}^{h T}+N_{I, 1} \mathbf{d}_{, 2}^{h T} & \hat{\mathbf{b}}_{12 I}^{h T}+\hat{\mathbf{b}}_{21 I}^{h T} \\
N_{I, 1} \mathbf{d}^{h T} & N_{I} \mathbf{b}_{1 I}^{h T} \\
N_{I, 2} \mathbf{d}^{h T} & N_{I} \mathbf{b}_{2 I}^{h T}
\end{array}\right]
$$

relates the variation of the strains

$$
\delta \boldsymbol{\varepsilon}^{h}=\sum_{I=1}^{n_{e n}} \mathbf{B}_{I}\left[\begin{array}{l}
\delta \mathbf{u}_{I} \\
\delta \boldsymbol{\beta}_{I}
\end{array}\right]
$$

to the variation of the nodal deformation vectors $\delta \mathbf{u}_{I}$ and the variation of nodal rotations $\delta \boldsymbol{\beta}_{I}$. The quantities $\hat{\mathbf{b}}_{\alpha \beta I}^{h}$, $\mathbf{b}_{\alpha I}^{h}$, the interpolation of the current director vector $\mathbf{d}^{h}$ and the nodal rotations $\boldsymbol{\beta}_{I}$ are defined in Sec. 7. The element stiffness matrix

$$
\mathbf{K}_{I K}^{e}=\int_{\Omega_{0}}\left(\mathbf{B}_{I}^{T} \mathbb{C} \mathbf{B}_{K}+\mathbf{G}_{I K}\right) \mathrm{d} \Omega
$$

requires the computation of the initial stress stiffness part $\mathbf{G}_{I K}$ of the element stiffness matrix, which is derived in [6]. The element residual vector is defined by

$$
\mathbf{f}_{I}^{e}=\int_{\Omega_{0}} \mathbf{B}_{I}^{T} \sigma \mathrm{d} \Omega-\mathbf{f}_{I}^{e, e x t} .
$$

Finally, the finite element approximation of the linearized weak form (19) reads

$$
\mathrm{L}[G(\mathbf{v}, \delta \mathbf{v})]=\bigcup_{e=1}^{n_{e l}} \sum_{I=1}^{n_{e n}} \sum_{K=1}^{n_{e n}}\left(\mathbf{f}_{I}^{e}+\mathbf{K}_{I K}^{e} \Delta \mathbf{v}_{K}\right)=\mathbf{f}+\mathbf{K} \widetilde{\mathbf{v}},
$$

where $\mathbf{f}$ is the global residuum vector, $\mathbf{K}$ the global stiffness matrix and $\widetilde{\mathbf{v}}$ collects the global deformations and rotations. 


\section{Computation of nodal basis systems for multi-patch geometries}

The interpolation of the director vector $\mathbf{D}$ needed for the description of the domain (13) and the interpolation of rotations require the definition of nodal basis systems. Patches which contain a kink along a line with $C^{0}$-continuity have to be split up into pieces along that line, as the director field has to be discontinuous in these regions. To avoid the classification if a kink occurs or not, patches are split up generally along lines of $C^{0}$-continuity. The necessary operations are trivial and given in [18]. No additional computational effort for the solution of the equilibrium equations turns up as the newly created control points are merged to one finite element node. To make use of the continuity of NURBS the nodal basis systems have to be chosen in a way that

$$
\mathbf{D}^{h}=\mathbf{A}_{3}^{h}=\sum_{I=1}^{n_{e n}} N_{I} \mathbf{A}_{3}^{(i, j, k)} \equiv \mathbf{N}
$$

holds and the resulting interpolated basis systems $\mathbf{A}_{i}^{h}$ are orthonormal, where the basis vectors $\mathbf{A}_{\alpha}^{h}$ can be chosen to simplify the imposition of boundary conditions or simply according to the lamina coordinate system proposed in [19]. A method to compute these nodal basis systems $\mathbf{A}_{i}^{(i, j, k)}$ for single patch geometries is proposed in [6]. This method is extended to multi-patch geometries in the following.

For every patch $k$ a separate system of equations is established and solved. $A_{m n}^{h}$ is the component $n$ of the basis vector $\mathbf{A}_{m}^{h}$. According to the tuple terminology defined in Sec. 2, $A_{m n}^{(i, j, k)}$ denotes the component $n$ of the basis vector $m$ at the control point $\mathbf{B}_{i j}^{k}$. For operations on patch level the function $f(i, j, k)$ is not bijective in general, which prohibits a summation over $I$. In all integration points $r$ ranging from 1 to

$$
n_{g p}^{k}=\left(\max \left(p_{1}^{k}, p_{2}^{k}\right)+1\right)^{2} \times n_{e l}^{k}
$$

the equations

$$
A_{m n}^{h}(r)=\sum_{i=1}^{n_{1}^{k}} \sum_{j=1}^{n_{2}^{k}} N_{I}(r) A_{m n}^{(i, j, k)}
$$

are established and collected line by line into one system of equations

$$
\overline{\mathbf{A}}_{m n}^{h}=\overline{\mathbf{N}}_{k} \overline{\mathbf{A}}_{m n}^{k}
$$

for every combination of $m$ and $n$. The matrix $\overline{\mathbf{N}}_{k}$ is independent of $m$ and $n$ and contains the basis functions $N_{I}(r)$ arranged in $n_{g p}^{k}$ rows and $n_{n p}^{k}$ columns. The vector $\overline{\mathbf{A}}_{m n}^{h}=\left[A_{m n}^{h}(r)\right]$ with $r=1, \ldots, n_{g p}^{k}$ is known and contains the component $n$ of the basis vector $\mathbf{A}_{m}^{h}$ at the integration point $r$. The unknown vector

$$
\overline{\mathbf{A}}_{m n}^{k}=\left[\left(A_{m n}^{(i, j, k)}\right)_{q}\right]
$$

where $q=1, \ldots, n_{n p}^{k}$ is a bijective function of the triple $(i, j, k)$, contains the component $n$ of the vector $m$ of the nodal basis system at control point $\mathbf{B}_{i j}^{k}$. In [6] it is shown, that a unique solution to (36) exists. Solving the system with the normal equation results in

$$
\overline{\mathbf{A}}_{m n}^{k}=\left(\overline{\mathbf{N}}_{k}^{T} \overline{\mathbf{N}}_{k}\right)^{-1} \overline{\mathbf{N}}_{k}^{T} \overline{\mathbf{A}}_{m n}^{h},
$$

which can be computed with standard solvers using multiple right hand sides to determine all nine components of the nodal basis systems. Finally, the nodal basis systems can be assembled from the solution of (38)

$$
\mathbf{A}_{m}^{(i, j, k)}=\left[\overline{\mathbf{A}}_{m n}^{k}(q)\right]_{n=1,2,3}
$$

and used for the interpolation of the director vector (46) and the interpolated basis systems (52).

Remark 1. This method is called Calculation of exact basis systems in [6], as a unique solution to (36) can always be found. This solution satisfies (38). Due to numerical reasons $\mathbf{N} \equiv \mathbf{D}^{h}$ is not fulfilled exactly but only close to the provided numerical precision. See [20] for the underlying mathematics. However, we use the term exactly to describe the interpolation of the director vector. 


\section{Differentiation between smooth and non-smooth nodes}

In common Lagrange-based finite element shell formulations with $C^{0}$-continuity between individual elements no well-defined distinction between nodes requiring three rotational degrees of freedom and those having none or negligible stiffness normal to the shell mid-surface is possible. Thus, nodes have to be classified by the user or drilling rotation stabilization has to be used. The concept proposed in Sec. 5 to compute nodal basis systems in combination with the higher inter-element continuity offered by the NURBS geometry description allows the definition of a unique criterion to distinguish between control points requiring six degrees of freedom and those having no rotational stiffness normal to the shell surface.

Kinks in NURBS geometries can only occur in $C^{0}$-continuous regions. On patch boundaries discontinuity prevails as open knot vectors are used. By using common finite element nodes for control points with corresponding locations, the geometry gets $C^{0}$-continuous at patch intersections. Patches with $C^{0}$-lines inside the domain have to be split up as described in Sec. 5.

Remark 2. Additional kinks can occur, if $p_{\alpha}^{k}$ control points coincide inside a patch of order $p_{\alpha}^{k}$. With these coinciding control points $C^{0}$-continuous points inside patches may occur. In these points the normal vector with respect to the shell surface is not defined. Thus using the normal vector instead of the director vector is not possible in these points. However, in the neighborhood of these points the director vector field cannot be exact. Those points only occur if the location of control points is modified manually and should be avoided on the design level.

All control points $\mathbf{B}_{i j}^{k}$ with

$$
\mathbf{X}^{(i, j, k)}=\mathbf{X}^{\left(i_{1}, j_{1}, k_{1}\right)} \quad i=1, \ldots, n_{1}^{k} \quad j=1, \ldots, n_{2}^{k} \quad k=1, \ldots, n_{p}
$$

are assigned to the same node $\mathbf{X}_{I}$ for the finite element analysis as the control point $\mathbf{B}_{i_{1} j_{1}}^{k_{1}}$ with $I=f\left(i_{1}, j_{1}, k_{1}\right)$, see Sec. 2. Accordingly, the vector of triples

$$
\mathbf{g}_{I}=\left\{(i, j, k) \mid \mathbf{X}^{(i, j, k)}=\mathbf{X}^{\left(i_{1}, j_{1}, k_{1}\right)} ; i=1, \ldots, n_{1}^{k} ; j=1, \ldots, n_{2}^{k} ; k=1, \ldots, n_{p}\right\}
$$

contains all $\# \mathbf{g}_{I}$ triples $(i, j, k)$ that are assigned to finite element node $I$, where $\# \mathbf{g}_{I}$ is the cardinality of the vector $\mathbf{g}_{I}$. All nodes $I$ which have only one triple $(i, j, k)$ assigned to them $\left(\# \mathbf{g}_{I}=1\right)$ are classified as smooth nodes. Thus, the reference global nodal basis system $\widetilde{\mathbf{A}}_{i I}$ is defined to be

$$
\widetilde{\mathbf{A}}_{i I}=\mathbf{A}_{i}^{(i, j, k)} .
$$

All finite element nodes $I$ with a cardinality $\# \mathbf{g}_{I} \geq 2$ require a distinction of cases. If

$$
\measuredangle\left(\mathbf{A}_{3}^{\mathbf{g}_{I}(l)}, \mathbf{A}_{3}^{\mathbf{g}_{I}(1)}\right) \leq c_{\text {angle }} \quad \forall l=1, \ldots, \# \mathbf{g}_{I}
$$

with the tolerance of angle $c_{\text {angle }}$ holds, then node $I$ is classified as smooth and

$$
\widetilde{\mathbf{A}}_{i I}=\mathbf{A}_{i}^{\mathbf{g}_{I}(l)}
$$

has to be chosen with a distinct value $l \in\left\{1, \ldots, \# \mathbf{g}_{I}\right\}$. If (43) does not hold, then node $I$ is classified as a node on kink. In this case the reference global nodal basis system is not required, respectively $\widetilde{\mathbf{A}}_{i I}$ can be chosen arbitrary. Depending on boundary conditions, a global Cartesian system $\widetilde{\mathbf{A}}_{i I}=\mathbf{e}_{i}$ or one of the nodal basis systems

$$
\widetilde{\mathbf{A}}_{i I}=\mathbf{A}_{i}^{\mathbf{g}_{l}(l)} \quad l \in\left\{1, \ldots, \# \mathbf{g}_{I}\right\}
$$

of the set of control points can be chosen. A reasonable value for the limit angle $c_{\text {angle }}$ is suggested in Sec. 8.1 with the help of a simple numerical example. The classification of nodes is needed in Sec. 7.3 to choose the appropriate equation for the transformation matrices $\mathbf{T}_{3 I}$, respectively $\mathbf{M}_{I}$. 


\section{Concepts for the interpolation of director vectors and description of rotations}

The main concern of this contribution lies in the treatment of the director vector and its variation with a special focus on non-smooth transitions between patches. The two presented concepts rely on a rotational formulation for the current director vector. The approach of computing the current director vector with difference vectors as proposed in [13] is not covered here. To introduce appropriate concepts for the description of rotations and director vectors at kinks, at first two possible concepts for the interpolation of the director vector in regular, smooth regions are sketched. Then the common treatment of kinks in standard $C^{0}$-Lagrange shell formulations is briefly summarized to show the shortcomings of current formulations. Consistent extensions to handle geometries with kinks are provided for both proposed concepts for the interpolation of the director vector. They emanate from the segregation of the interpolation of the director and the interpolation of the rotations and thus combine the advantages of both traditional concepts.

\subsection{Interpolation of the director vector and its variation}

The reference director $\mathbf{D}$ introduced in (13) to describe the initial and the deformed domain is generally interpolated by

$$
\mathbf{D}^{h}=\sum_{I=1}^{n_{e n}} N_{I} \mathbf{D}_{I},
$$

where $N_{I}$ are the NURBS basis functions defined in (8). The discrete nodal directors $\mathbf{D}_{I}$ will be defined later on in Sec. 7.3. The derivations with respect to the local Cartesian system

$$
\mathbf{D}_{, \alpha}^{h}=\sum_{I=1}^{n_{e n}} N_{I, \alpha} \mathbf{D}_{I}
$$

are computed with the derivatives of the NURBS basis functions defined in (25). In the following $\mathbf{A}_{i I}$ and $\mathbf{a}_{i I}$ are discrete nodal basis systems in the reference configuration and in the current configuration. In rotational shell formulations the current director vector is computed by an orthogonal rotation $\mathbf{d}=\mathbf{R D}$. In general several strategies for the computation of the current rotational state $\mathbf{R}^{i}$ are possible, see detailed discussions in [21, 22]. Multiplicative update schemes

$$
\mathbf{R}^{i}=\mathbf{R}(\Delta \omega) \mathbf{R}^{i-1}
$$

as proposed in [4] yield a non-symmetric global stiffness matrix $\mathbf{K}$, see [22]. Here, an additive update method using a vector-like parametrization of rotations as proposed by [23] is used. This ensures the symmetry of the global stiffness matrix, simplifies the implementation and needs less storage. The axial vector of the rotation is updated according to

$$
\omega^{i}=\omega^{i-1}+\Delta \omega
$$

where $\omega^{i-1}$ is the value from the last iteration step and $\Delta \omega$ is the increment of the last iteration. The rotation tensor

with

$$
\mathbf{R}=\mathbf{1}+\frac{\sin \omega}{\omega} \boldsymbol{\Omega}+\frac{1-\cos \omega}{\omega^{2}} \boldsymbol{\Omega}^{2}
$$

$$
\boldsymbol{\Omega}=\operatorname{skew} \boldsymbol{\omega}=\left[\begin{array}{ccc}
0 & -\omega_{3} & \omega_{2} \\
\omega_{3} & 0 & -\omega_{1} \\
-\omega_{2} & \omega_{1} & 0
\end{array}\right] \quad \omega=\|\boldsymbol{\omega}\|
$$

is computed with the Rodrigues' formula using the updated rotation vector $\omega^{i}$. As (50) is singular for $\omega=k \cdot 2 \pi$ with $k=1,2, \ldots$, a combined strategy proposed by [22] has to be used for finite rotations. After each load step a multiplicative update is conducted to preclude singularities. Within the iterations of each load step the additive update ensures the symmetry of the global stiffness matrix. For a more recent overview on the treatment of finite rotations, see [24]. Depending on whether interpolated values $\omega^{h}$ or nodal values $\omega_{I}$ are inserted into (50) the interpolated rotation tensor $\mathbf{R}^{h}$ or nodal rotation tensors $\mathbf{R}_{I}$ arise. Analogously current basis systems are computed by

$$
\mathbf{a}_{i}^{h}=\mathbf{R}^{h} \mathbf{A}_{i}^{h} \quad \mathbf{A}_{i}^{h}=\sum_{I=1}^{n_{e n}} N_{I} \mathbf{A}_{i I},
$$

respective $\mathbf{a}_{i I}=\mathbf{R}_{I} \mathbf{A}_{i I}$.

When $\mathbf{d}$ and its variation $\delta \mathbf{d}$ need to be interpolated, in general two possibilities arise. 
Concept a) Interpolation after rotation. The first possibility is to rotate each discrete value $\mathbf{D}_{I}$ with its corresponding rotation tensor $\mathbf{R}_{I}$ and interpolate afterwards. This results in

$$
\mathbf{d}^{h}=\sum_{I=1}^{n_{e n}} N_{I} \mathbf{d}_{I} \quad \mathbf{d}_{, \alpha}^{h}=\sum_{I=1}^{n_{e n}} N_{I, \alpha} \mathbf{d}_{I} \quad \mathbf{d}_{I}=\mathbf{R}_{I} \mathbf{D}_{I}
$$

for the interpolation of the current director vector and for its derivation. The variation of the shell strains requires the variation of the current director vector, whose interpolation is akin to (53) defined as

$$
\delta \mathbf{d}^{h}=\sum_{I=1}^{n_{e n}} N_{I} \delta \mathbf{d}_{I} \quad \delta \mathbf{d}_{, \alpha}^{h}=\sum_{I=1}^{n_{e n}} N_{I, \alpha} \delta \mathbf{d}_{I}
$$

with $\delta \mathbf{d}_{I}=\mathbf{T}_{I} \delta \boldsymbol{\beta}_{I}$. In smooth areas the two virtual nodal rotations $\delta \beta_{\alpha I}$ are defined around the two vectors $\mathbf{a}_{\alpha I}=\mathbf{R}_{I} \mathbf{A}_{\alpha I}$ of the current nodal basis system. The matrix $\mathbf{T}_{I}$ is a function of the current nodal director vector $\mathbf{d}_{I}$, the vectors $\mathbf{a}_{\alpha I}$ and the rotations $\boldsymbol{\beta}_{I}$.

Concept b) Rotation after interpolation. The second possibility is to compute the reference director vector in every integration point and rotate this vector with a rotation tensor $\mathbf{R}^{h}$ defined at the integration point. $\mathbf{R}^{h}$ is computed using (50) and (51) with the interpolated rotations $\omega^{h}$. The resulting current director vector and its derivation are defined as

$$
\mathbf{d}^{h}=\mathbf{R}^{h} \mathbf{D}^{h} \quad \mathbf{d}_{, \alpha}^{h}=\mathbf{R}_{, \alpha}^{h} \mathbf{D}^{h}+\mathbf{R}^{h} \mathbf{D}_{, \alpha}^{h},
$$

where the inextensibility condition $\left\|\mathbf{d}^{h}\right\|=\left\|\mathbf{D}^{h}\right\|$ is exactly fulfilled in every integration point. The consistent variation of the first equation of (55) results in

$$
\delta \mathbf{d}^{h}=\mathbf{T} \delta \boldsymbol{\beta}^{h},
$$

where the matrix $\mathbf{T}$ is defined at the integration points and is a function of the interpolated current director vector $\mathbf{d}^{h}=\mathbf{a}_{3}^{h}$, the vectors $\mathbf{a}_{\alpha}^{h}$ of the interpolated, orthonormal basis systems and the interpolated rotations $\boldsymbol{\beta}^{h}$. Subsequently, the variation of the rotations is interpolated with

$$
\delta \boldsymbol{\beta}^{h}=\sum_{I=1}^{n_{e n}} N_{I} \mathbf{M}_{I} \delta \boldsymbol{\beta}_{I}
$$

where the matrix $\mathbf{M}_{I}$ accomplishes a transformation of the nodal rotations $\delta \boldsymbol{\beta}_{I}$, which are defined around the nodal basis systems $\mathbf{a}_{\alpha I}$, to rotations around the interpolated basis vectors $\mathbf{a}_{\alpha}^{h}$. The derivation of $\delta \mathbf{d}^{h}$ is more involved and reads

$$
\delta \mathbf{d}_{, \alpha}^{h}=\mathbf{T}_{, \alpha} \sum_{I=1}^{n_{e n}} N_{I} \mathbf{M}_{I} \delta \boldsymbol{\beta}_{I}+\mathbf{T} \sum_{I=1}^{n_{e n}}\left(N_{I, \alpha} \mathbf{M}_{I}+N_{I} \mathbf{M}_{I, \alpha}\right) \delta \boldsymbol{\beta}_{I} .
$$

All required matrices are given in Sec. 7.3.

Remark 3. In NURBS-based isogeometric analysis it is also possible to use the normal vector $\mathbf{N}$ with regard to the shell mid-surface instead of the interpolated reference director vector $\mathbf{D}^{h}$. Additional studies conducted by the authors do not show significant improvements over the interpolation with exactly calculated nodal basis systems. To avoid the need to compute the second spatial derivatives within the assembly of the stiffness matrix here the reference director is used. Furthermore numerical problems on points with $C^{0}$-continuity within patches are avoided, see remark 2.

\subsection{Concepts for $C^{0}$-Lagrange shell elements for geometries with kinks}

Shell formulations based on traditional $C^{0}$-Lagrange basis functions use commonly concept $a$ ), where the rotated nodal director vectors are interpolated. In [4] it is noted, that although other concepts were tested to be slightly superior, this concept is sufficiently accurate for the description of rotations in shell formulations with linear Lagrange basis functions. An important choice for $C^{0}$-Lagrange elements is the definition of nodal directors $\mathbf{D}_{I}$, see a detailed discussion in [1]. Using common global directors at each node while preserving the orthogonality between surface and director vector is only possible for $C^{1}$-continuous surfaces. Thus, two common concepts are used to circumvent the need for $C^{1}$-continuity, violating either continuity or orthogonality. Figs. 1 and 2 illustrate both concepts. 


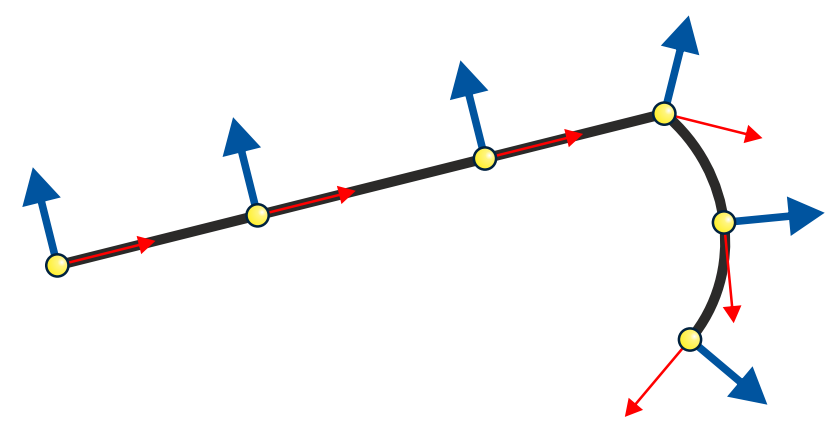

Figure 1: Unique global nodal basis systems for $C^{0}$-Lagrange shell elements.

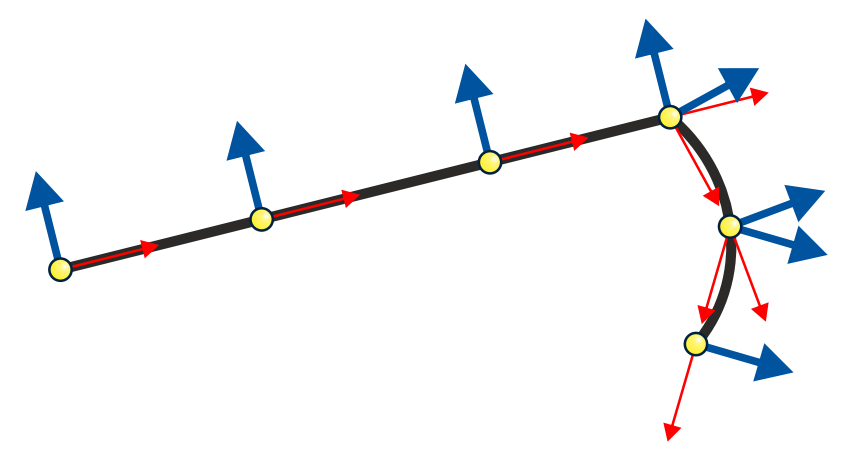

Figure 2: Element wise defined nodal basis systems for $C^{0}$-Lagrange shell elements.

a1) Unique global director vectors. For the modeling of smooth surfaces global nodal directors $\mathbf{D}_{I}$ are chosen commonly as normalized algebraic average of all neighboring elements. All discontinuities - no matter if they arise from the faceting of smooth surfaces or from intentionally modeled kinks - are treated the same way as if $C^{1}$-continuity would prevail. When a node is located at a kink the orthogonality of the director vector is violated in general, see Fig. 1. Drilling degrees of freedom can be avoided with this concept as nodal rotations are defined around unique nodal basis systems.

a2) Element wise defined director vectors. For geometries containing kinks and complex intersections of several shell mid-surfaces it is desirable to avoid the determination of a common director vector. By using nodal directors which are defined individually for every element, the violation of the orthogonality of the director vector can be completely avoided. But this leads to a summation of rotations around differing nodal basis systems for one control point when computing faceted geometries or geometries with kinks, see Fig. 2. At smooth transitions the nodal basis systems have to be aligned, which is not possible in general, e.g. at intersections of three patches in one point, see Fig. 3. A third rotational - also called drilling - degree of freedom is introduced at kinks to couple rotations consistently. The stiffness against this additional rotation is caused by the intersection of the surfaces in different angles. Depending on the geometrical situation drilling rotation stiffness can be small in comparison to the two main rotational stiffness and thus numerical problems arise. This is usually avoided by adding artificial stiffness against drilling rotations (drilling rotation stabilization). The usage of element wise directors with three rotational degrees of freedom for all nodes in combination with drilling rotation stabilization is common in industrial applications [1]. Thus, the need for a manual classification of nodes is avoided. But the numerical results are influenced by the choice of drilling rotation stabilization parameters.

\subsection{Concepts for isogeometric shell elements for geometries with kinks}

The higher continuity of NURBS-based isogeometric analysis offers new possibilities to treat geometries with kinks more appropriate. Between the individual patches no continuity is at hand as each patch is defined with its own set of control points. By coupling the deformations of control points with corresponding locations $C^{0}$-continuity 


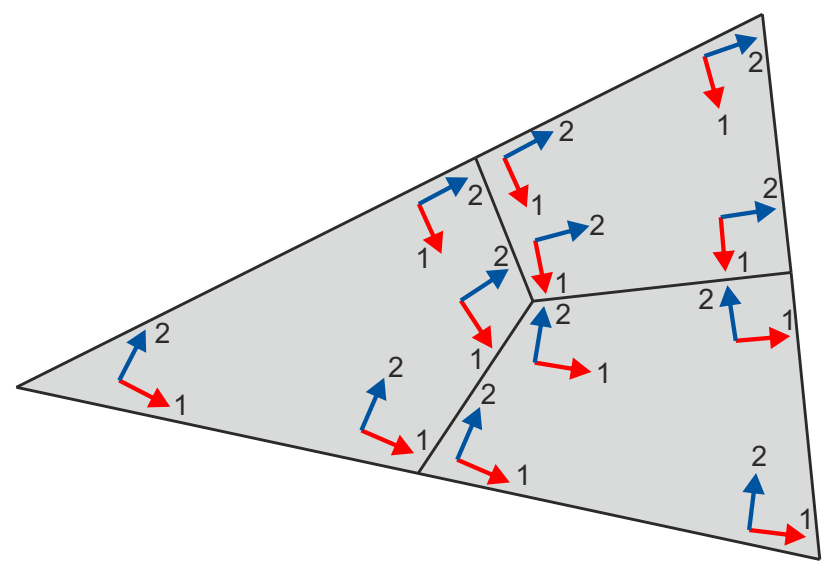

Figure 3: Intersection of three patches at one point: Orthonormal basis systems computed from the parametric derivatives in the four vertices of each patch. A choice where all basis systems are aligned is not possible.

of deformations is ensured. The possibilities offered by the high continuity of NURBS are fully exploited with the proposed concepts, as transitions between patches can be uniquely classified (see Sec. 6) to be smooth or non-smooth and then accordingly treated. In the following both concepts for the interpolation of the current director vector are consistently extended to handle geometries with kinks. Fig. 4 illustrates the usage of two distinct sets of nodal basis systems, which is a decisive part of these extensions.

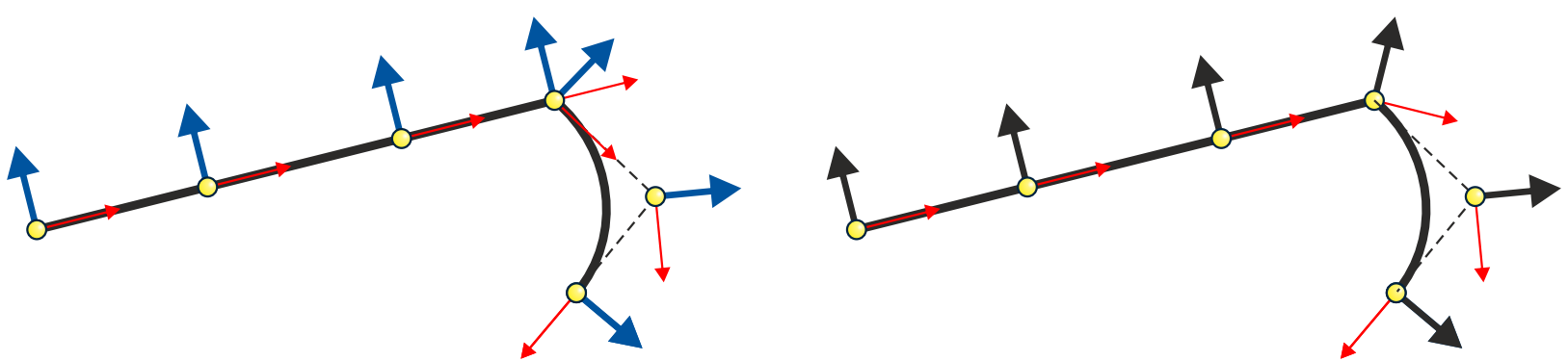

Figure 4: Proposed concept for isogeometric shell elements: Patch wise defined nodal basis system (left) for the interpolation of the director and global defined nodal basis system (right) for the interpolation of rotations.

Concept a) Interpolation after rotation. A consistent treatment of kinks is possible with the patch wise defined nodal basis systems $\mathbf{A}_{m}^{(i, j, k)}$ computed in Sec. 5. Within each patch at least $C^{1}$-continuity has to prevail. If this holds, the application of concept a2) described in Sec. 7.2 produces a continuous interpolation of the director vector within each patch and a discontinuous interpolation on kinks. Thus, the orthogonality of the director vector is ensured. The nodal directors

$$
\mathbf{D}_{I}=\mathbf{A}_{3}^{(i, j, k)}
$$

are attained with $(i, j, k)=g(I, e)$ for every node $I$ of element $e$. Thus the standard interpolation of the reference director vector

$$
\mathbf{D}^{h}=\sum_{I=1}^{n_{e n}} N_{I} \mathbf{D}_{I}
$$

can be used and $\mathbf{N}=\mathbf{D}^{h}$ holds, where $\mathbf{N}$ is the normal vector with respect to the mid-surface. The variated nodal director vectors $\delta \mathbf{d}_{I}$ required in (54) are computed with

$$
\delta \mathbf{d}_{I}=\mathbf{T}_{I} \delta \boldsymbol{\beta}_{I}=\mathbf{W}_{I}^{T} \mathbf{H}_{I} \mathbf{T}_{3 I} \delta \boldsymbol{\beta}_{I},
$$


where the matrices $\mathbf{W}_{I}=$ skew $\mathbf{d}_{I}$ and

$$
\mathbf{H}_{I}=\mathbf{1}+\frac{1-\cos \omega}{\omega^{2}} \boldsymbol{\Omega}+\frac{\omega-\sin \omega}{\omega^{3}} \mathbf{\Omega}^{2}
$$

are discussed in detail in [25]. For (62) the definitions of (51) are used with nodal values $\omega_{I}$. The increment of the rotation vector needed for the update of the rotation matrix $\mathbf{R}_{I}$ given in (49) is computed by

$$
\Delta \omega_{I}=\mathbf{T}_{3 I} \Delta \beta_{I}
$$

Depending on the classification of the node I (see Sec. 6) the matrix $\mathbf{T}_{3 I}$ is defined as:

- Nodes on kinks are assigned three rotational degrees of freedom to allow a consistent coupling of rotations. As long as the angle of the kink does not approach zero, stiffness against drilling rotations is provided by the intersection of the surfaces. Here the axial vector of the rotation $\delta \omega_{I}$ is kept as rotational parameter. Thus no transformation is necessary and the matrix

$$
\mathbf{T}_{3 I}=\mathbf{1}
$$

is defined as a $3 \times 3$ unity matrix. This results in three nodal rotations $\delta \beta_{i I}$ around a global Cartesian basis system.

- Smooth nodes require only two rotational degrees of freedom. Stiffness against drilling rotations does not arise. Thus the transformation matrix $\mathbf{T}_{3 I}$ is defined as

$$
\mathbf{T}_{3 I}=\left[\begin{array}{ll}
\widetilde{\mathbf{a}}_{1 I} & \widetilde{\mathbf{a}}_{2 I}
\end{array}\right]
$$

where the vectors $\widetilde{\mathbf{a}}_{\alpha I}=\mathbf{R}_{I} \widetilde{\mathbf{A}}_{\alpha I}$ are computed from the global nodal basis system $\widetilde{\mathbf{A}}_{\alpha I}$ for node $I$. Two nodal rotations $\delta \beta_{\alpha I}$ arise.

With the nodal values of $\delta \mathbf{d}^{h}$ given in (61) at hand the quantities $\hat{\mathbf{b}}_{\alpha \beta I}^{h}$ and $\mathbf{b}_{\alpha I}^{h}$ required in (28) can be computed by

$$
\hat{\mathbf{b}}_{\alpha \beta I}^{h}=N_{I, \alpha} \mathbf{T}_{I}^{T} \mathbf{x}_{, \beta}^{h}
$$

and

$$
\mathbf{b}_{\alpha I}^{h}=\mathbf{T}_{I}^{T} \mathbf{x}_{, \alpha}^{h} .
$$

For the linearization and the resulting initial stress stiffness matrix $\mathbf{G}_{I K}$ we refer to [26].

Remark 4. The presented method for concept a) differs only in one small point from the standard procedure applied in common $C^{0}$ Lagrange shell formulations. The director vector is interpolated with patch wise defined nodal vectors, whereas the rotations are described around globally defined nodal basis systems. This small alteration allows the usage of an arbitrary parametrization of neighboring patches. The parametric directions do not have to match, which simplifies meshing dramatically in comparison to standard Lagrange-based shell formulations.

Concept b) Rotation after interpolation. A consistent treatment of kinks is ensured by using a $C^{0}$-continuous description of rotations with the global nodal basis systems $\widetilde{\mathbf{A}}_{i I}$ and an interpolation of director vectors, which is discontinuous to preserve the orthogonality. Using concept $b$ ) to interpolate the current director vector allows the usage of two independent interpolations, one for the reference director vector (46) and another one for the rotations (57). Both interpolations use the same basis functions and thus initially exhibit the same continuity as the geometry. The nodal basis systems $\mathbf{A}_{m}^{(i, j, k)}$ interpolate the normal vector with respect to the mid-surface exactly using (59) and (60). The smoothness of the geometry is replicated as desired. To achieve a consistent coupling of the rotations, intersecting patches use common rotational degrees of freedom $\delta \boldsymbol{\beta}_{I}$ with one arbitrary common global nodal basis system $\widetilde{\mathbf{A}}_{i I}$ for each set of corresponding control points. Thus, $C^{0}$-continuity for the variation of the axial vector of the rotations

$$
\delta \boldsymbol{\omega}^{h}=\mathbf{T}_{3} \delta \boldsymbol{\beta}^{h}=\mathbf{T}_{3} \sum_{I=1}^{n_{e n}} N_{I} \mathbf{M}_{I} \delta \boldsymbol{\beta}_{I}
$$


is attained and the rotations $\boldsymbol{\beta}$ are consistently coupled under consideration of the differing orientations of nodal basis systems. The matrix

$$
\mathbf{T}_{3}=\left[\begin{array}{ll}
\mathbf{a}_{1}^{h} & \mathbf{a}_{2}^{h}
\end{array}\right] \quad \text { with } \quad \mathbf{a}_{\alpha}^{h}=\mathbf{R} \mathbf{A}_{\alpha}^{h}=\mathbf{R} \sum_{I=1}^{n_{e n}} \mathbf{A}_{\alpha}^{(i, j, k)}
$$

contains the interpolated current basis systems, around which the rotations $\delta \boldsymbol{\beta}_{\alpha}$ in the integration points are defined. The updates of the rotation matrices $\mathbf{R}$ and $\mathbf{R}_{I}$ are conducted according to (49) with the increment of the rotation vector in the integration point and the increment of the nodal rotation vector

$$
\begin{aligned}
\Delta \omega^{h} & =\mathbf{T}_{3} \sum_{I=1}^{n_{e n}} N_{I} \mathbf{M}_{I} \Delta \boldsymbol{\beta}_{I} \\
\Delta \omega_{I} & =\mathbf{T}_{3 I} \Delta \boldsymbol{\beta}_{I} .
\end{aligned}
$$

Depending on the classification of the node $I$ (see Sec. 6), the matrix $\mathbf{M}_{I}$ and its derivatives are defined as:

- Nodes on kinks need three rotational degrees of freedom to describe the rotations consistently. The three nodal rotations $\Delta \beta_{i I}$ are transformed with the matrix

$$
\mathbf{M}_{I}=\left[\begin{array}{lll}
\mathbf{a}_{1}^{h} \cdot \widetilde{\mathbf{a}}_{1 I} & \mathbf{a}_{1}^{h} \cdot \widetilde{\mathbf{a}}_{2 I} & \mathbf{a}_{1}^{h} \cdot \widetilde{\mathbf{a}}_{3 I} \\
\mathbf{a}_{2}^{h} \cdot \widetilde{\mathbf{a}}_{1 I} & \mathbf{a}_{2}^{h} \cdot \widetilde{\mathbf{a}}_{2 I} & \mathbf{a}_{2}^{h} \cdot \widetilde{\mathbf{a}}_{3 I}
\end{array}\right]
$$

from the current global nodal basis system $\widetilde{\mathbf{a}}_{i I}=\mathbf{R}_{I} \widetilde{\mathbf{A}}_{i I}$ to the current interpolated basis system $\mathbf{a}_{i}^{h}$ in the integration point. Virtual rotations around the normal vector in the integration point do not cause stiffness and can thus simply be omitted. The transformation matrix (71) allows the use of arbitrary basis systems for control points on kinks. The derivative of $\mathbf{M}_{I}$ is

$$
\mathbf{M}_{I, \alpha}=\left[\begin{array}{llll}
\mathbf{a}_{1, \alpha}^{h} \cdot \widetilde{\mathbf{a}}_{1 I} & \mathbf{a}_{1, \alpha}^{h} \cdot \widetilde{\mathbf{a}}_{2 I} & \mathbf{a}_{1, \alpha}^{h} \cdot \widetilde{\mathbf{a}}_{3 I} \\
\mathbf{a}_{2, \alpha}^{h} \cdot \widetilde{\mathbf{a}}_{1 I} & \mathbf{a}_{2, \alpha}^{h} \cdot \widetilde{\mathbf{a}}_{2 I} & \mathbf{a}_{2, \alpha}^{h} \cdot \widetilde{\mathbf{a}}_{3 I}
\end{array}\right]
$$

- Nodes in smooth regions require only two rotational degrees of freedom. Stiffness against drilling rotations does not arise. In this case the matrix

$$
\mathbf{M}_{I}=\left[\begin{array}{ll}
\mathbf{a}_{1}^{h} \cdot \widetilde{\mathbf{a}}_{1 I} & \mathbf{a}_{1}^{h} \cdot \widetilde{\mathbf{a}}_{2 I} \\
\mathbf{a}_{2}^{h} \cdot \widetilde{\mathbf{a}}_{1 I} & \mathbf{a}_{2}^{h} \cdot \widetilde{\mathbf{a}}_{2 I}
\end{array}\right]
$$

transforms two nodal rotations $\Delta \beta_{\alpha I}$ from the current global nodal basis system $\widetilde{\mathbf{a}}_{i I}$ to the current interpolated basis system $\mathbf{a}_{i}^{h}$ at the integration point. Here the nodal basis systems have to be chosen aligned to the physcial domain, as computed in Sec. 5, since only two rotations are considered. The derivative of $\mathbf{M}_{I}$ is

$$
\mathbf{M}_{I, \alpha}=\left[\begin{array}{ccc}
\mathbf{a}_{1, \alpha}^{h} \cdot \widetilde{\mathbf{a}}_{1 I} & \mathbf{a}_{1, \alpha}^{h} \cdot \widetilde{\mathbf{a}}_{2 I} \\
\mathbf{a}_{2, \alpha}^{h} \cdot \widetilde{\mathbf{a}}_{1 I} & \mathbf{a}_{2, \alpha}^{h} \cdot \widetilde{\mathbf{a}}_{2 I}
\end{array}\right]
$$

The variation of the current director vector (56) and its derivation (58) require furthermore the defintion of the matrix $\mathbf{T}$ and its derivation

$$
\mathbf{T}=\mathbf{W}^{T} \mathbf{H} \mathbf{T}_{3} \quad \mathbf{T}_{, \alpha}=\mathbf{W}_{, \alpha}^{T} \mathbf{H} \mathbf{T}_{3}+\mathbf{W}^{T} \mathbf{H}_{, \alpha} \mathbf{T}_{3}+\mathbf{W}^{T} \mathbf{H} \mathbf{T}_{3, \alpha}
$$

with $\mathbf{W}=$ skew $\mathbf{d}$ and $\mathbf{T}_{3}$ given in (69). The derivatives of these matrices are straightforward and thus omitted. The derivation of

is more involved and reads

$$
\mathbf{H}=\mathbf{1}+\frac{1-\cos \omega}{\omega^{2}} \boldsymbol{\Omega}+\frac{\omega-\sin \omega}{\omega^{3}} \mathbf{\Omega}^{2}
$$

$$
\begin{array}{rlrl}
\mathbf{H}_{, \alpha} & =h_{1} \boldsymbol{\Omega}+h_{2} \boldsymbol{\Omega}_{, \alpha}+h_{3} \boldsymbol{\Omega}^{2}+h_{4}\left(\boldsymbol{\Omega}_{, \alpha} \boldsymbol{\Omega}+\mathbf{\Omega}_{, \alpha}\right) \\
h_{1} & =\omega_{, \alpha} \frac{\omega \sin \omega-2(1-\cos \omega)}{\omega^{3}} & h_{2}=\frac{1-\cos \omega}{\omega^{2}} \\
h_{3} & =\omega_{, \alpha} \frac{3 \sin \omega-\omega \cos \omega-2 \omega}{\omega^{4}} & h_{4} & =\frac{\omega-\sin \omega}{\omega^{3}} .
\end{array}
$$


Finally the quantities

$$
\hat{\mathbf{b}}_{\alpha \beta I}^{h}=\hat{\mathbf{T}}_{I, \alpha}^{T} \mathbf{x}_{, \beta}^{h} \quad \hat{\mathbf{T}}_{I, \alpha}=\mathbf{T}_{, \alpha} \mathbf{M}_{I} N_{I}+\mathbf{T}\left(N_{I, \alpha} \mathbf{M}_{I}+N_{I} \mathbf{M}_{I, \alpha}\right)
$$

and

$$
\mathbf{b}_{\alpha I}^{h}=\mathbf{M}_{I}^{T} \mathbf{T}^{T} \mathbf{x}_{, \alpha}^{h}
$$

required in (28) can be computed. For more details including the complete derivation and linearization as well as for the resulting initial stress stiffness matrix $\mathbf{G}_{I K}$ we refer to [6].

Remark 5. The transformation matrix proposed in [6] contains a statical condensation, which may cause numerical problems for strongly curved elements in coarse meshes. Here this transformation is omitted, as the resulting deformations are almost identical.

\subsection{Remark on varying results between both proposed concepts}

The numerical examples in Sec. 8 show that the converged deformations computed with both concepts slightly differ for nonlinear computations, whereas they are equivalent for linear computations. The reasons for this behavior shall be investigated in the following with the help of the formulae provided above. Concept a) relies on rotations defined around nodal basis systems. Nodes on kinks are assigned three global rotations. In the linear case $\mathbf{H}_{I}=\mathbf{1}$ holds and thus the third column of $\mathbf{W}_{I}^{T} \mathbf{H}_{I} \mathbf{T}_{3 I}$ reads $\mathbf{a}_{3 I} \times \mathbf{a}_{3 I}=\mathbf{0}$. Stiffness arises only against rotations around the two local rotational axes $\mathbf{a}_{\alpha I}$. In the nonlinear case the third column of $\mathbf{W}_{I}^{T} \mathbf{H}_{I} \mathbf{T}_{3 I}$ has non-zero entries. Thus, stiffness against rotations around all three nodal basis vectors arises, although the integration points are located in smooth regions. In concept $b$ ), the rotations are described around interpolated basis systems in the integration points. The rotations of the nodes are just needed as common rotational axes. Integration points are always in smooth regions and thus two rotations are sufficient in the integration points. The transformation matrix $\mathbf{M}_{I}$ ensures that only the rotations suitable to the basis system in the integration point under consideration are inherited from nodes which are located on kinks and require three rotations. The matrix $\mathbf{T}=\mathbf{W}^{T} \mathbf{H} \mathbf{T}_{3}$ has only two columns. Thus, only stiffness against rotations around the two local rotational axes $\mathbf{a}_{\alpha I}$ can arise. However, for all computed examples the difference between the converged deformations for the two proposed concepts is very small, especially in comparison to the effects of other simplifications.

\section{Numerical examples}

The capability of the presented shell formulations for the computation of single patch domains is shown in [6]. There the convergence behavior is tested with linear and nonlinear computations of common benchmark examples and with a newly defined benchmark with strong changes in curvature. A comparison between computations with concept $a$ ) and concept $b$ ) for the description of rotations is given. The converged deformations computed with very fine discretizations are equivalent in each case for all presented examples. Differences occur in the convergence behavior of deformation results, i.e. the decrease of the deformation error for mesh refinement respectively order elevation. For weakly curved shells and shells with constant curvatures only marginal differences in deformation results occur, and the convergence behavior is similar for both concepts. However, for arbitrarily curved shells concept $b$ ) proved to yield superior deformation results for coarse meshes as well as superior convergence behavior in comparison to concept $a$ ). Only concept $b$ ) yields correct convergence behavior for order elevation with $k$-refinement. The superior performance of concept $b$ ) is due to its following properties, see [6]. The exact interpolation of the reference director vector and the subsequent rotation of this vector ensure a correct interpolation of the kinematic assumptions even for the coarsest mesh. Differing orientations between interpolated basis systems and nodal basis systems are taken into account for the interpolation of rotations.

The numerical examples in the following focus on the treatment of multi-patch domains with kinks and sharp folds. The first numerical example has the intention to find an indicative limit angle for the distinction between smooth patch transitions and kinks. The plane gravity loaded L-shaped plate tests the behavior of the shell formulations at smooth intersections with a change in the derivatives. The channel section beam under point load shows the ability of the formulations to capture large rotations and to transfer rotations at the intersections consistently. Reference computations with the isogeometric shell elements of the commercial finite element code LS-DYNA are provided. Complex intersections are tested with the pyramid under surface load. The partly clamped hyperbolic paraboloid with 


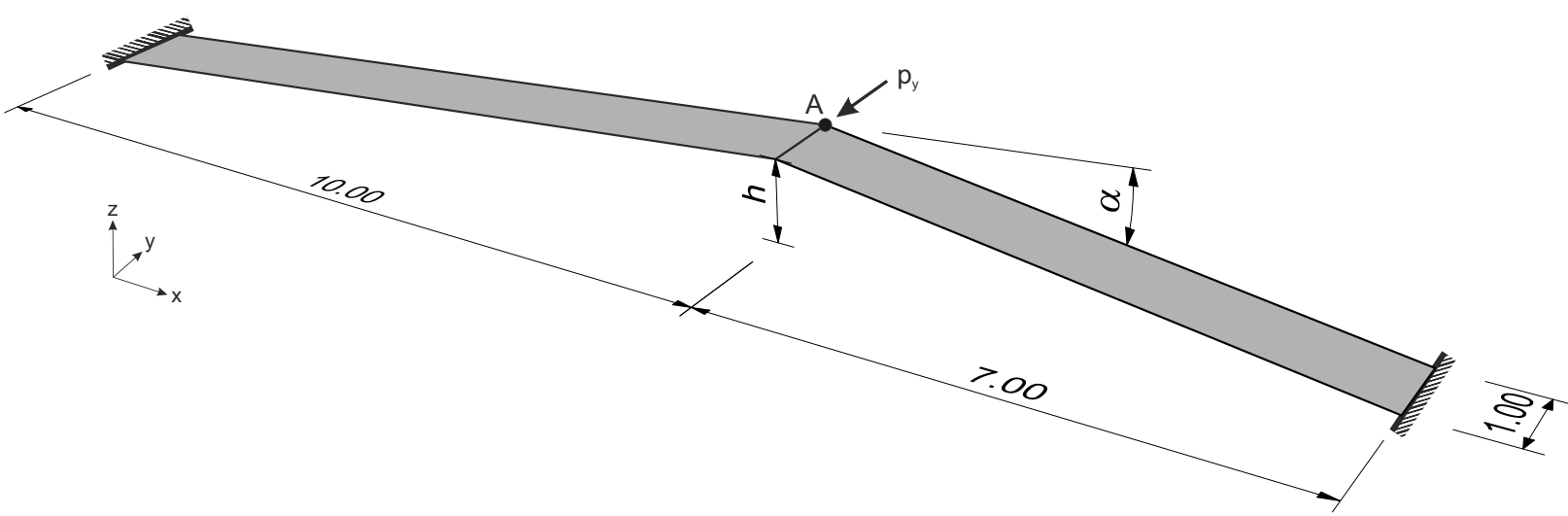

Figure 5: Simple shallow truss structure: System sketch.

stiffeners combines a curved geometry with complex intersections. With the free form surface with arbitrary curvature and kink the quality of the deformation results for geometries with large and arbitrary curvature in combination with kinks is compared between the two concepts. The complex geometry of this example allows to study the influence of the limit intersection angle in the linear and nonlinear case. All computations are performed with a St. VenantKirchhoff material model. For the post-processing of the numerical examples parts of the integrated design-throughanalysis workflow proposed in $[8,27]$ are used.

\subsection{Basic numerical example: Simple shallow truss structure}

With the help of the shallow truss structure depicted in Fig. 5 an indicative value for the angle of tolerance $c_{\text {angle }}$ is proposed. The study of this shallow structure with a varied height allows the investigation of the transition between shallow structures with kink and smooth structures. A limit intersection angle for the classification of treatment as smooth transition (two rotational degrees of freedom) or as transition with kink (three rotational degrees of freedom) has to be found. For intersection angles approaching zero the condition of the stiffness matrix deteriorates, as the stiffness against the third rotational degree of freedom is generated by the intersection of the surfaces. However, the treatment of an intersection - although with small intersection angle - as smooth does not represent the correct physical behavior and is thus erroneous. A structure composed of two plane patches is chosen, as in this case minimal stiffness against drilling rotations arises. The Young's modulus is $2.1 \cdot 10^{5}$ with a Poisson's ratio $v=0.3$. The load $p_{y}=1.0$ is applied as a line load over the whole apex. All degrees of freedom are held at both clamped ends. Computations are linear. The height of the structure is varied between $h=0.001$ and $h=1.0$. The resulting angle $\alpha$ is computed by

$$
\alpha=\arctan (h / 10)+\arctan (h / 7)
$$

and thus takes values between $0.01391^{\circ}$ and $13.8407^{\circ}$. The deformations of point $\mathbf{A}$ at the apex are compared. The ratio between the maximum and the minimum diagonal entry is used as an estimate for the condition of the stiffness matrix. Fig. 7 provides the associated error of the calculation as smooth transition relative to the mechanical correct calculation with drilling degrees of freedom using the same discretization. In Figs. 8 and 9 corresponding results are given for an $h$-refinement study with order $p=4$. The results of both studies are akin, thus the conclusion is valid for both kinds of refinement. The condition estimate for calculations with six degrees of freedom at the apex grows exponentially for a decreasing intersection angle, whereas the deformation error decreases exponentially. The condition of calculations with five degrees of freedoms stays more or less constant. For constant intersection angles the condition deteriorates and the error decreases with rising order of NURBS respective mesh refinement. Depending on the desired accuracy a limit intersection angle between $1^{\circ}$ and $3^{\circ}$ seems to be reasonable. The authors suggest using a value of $2^{\circ}$. It should be mentioned, that for this example computing a kink with an angle $\alpha=2^{\circ}$ as if it would be smooth, leads only to a very small relative error in the range of $10^{-4}$ with respect to computations using the correct classification. This alteration of the results is negligible in comparison to the effects of drilling rotation stabilization. A broader set of different examples should be considered to attain a generally valid limit intersection angle. The proposed value of $2^{\circ}$ is intended to be a rough indicative value. 


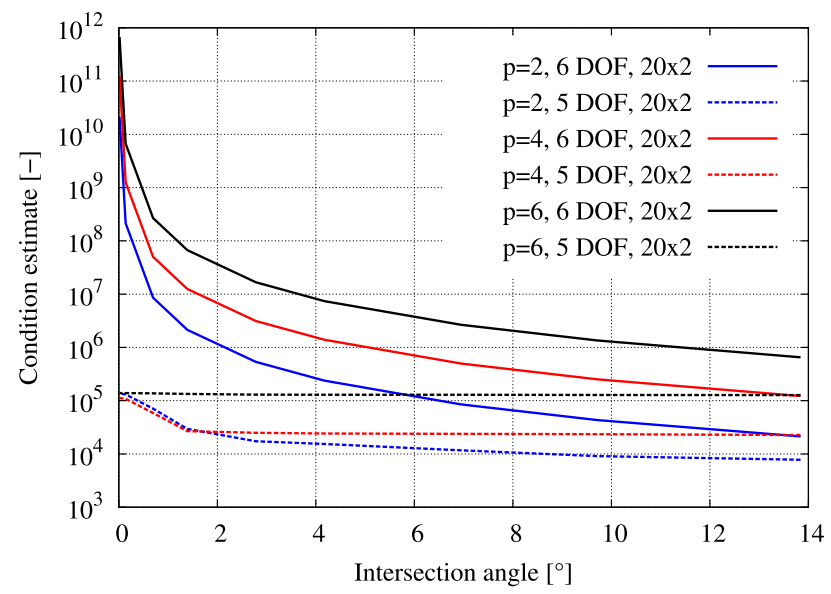

Figure 6: Simple shallow truss structure: Condition estimate for $k$-refinement and $20 \times 2$ elements.

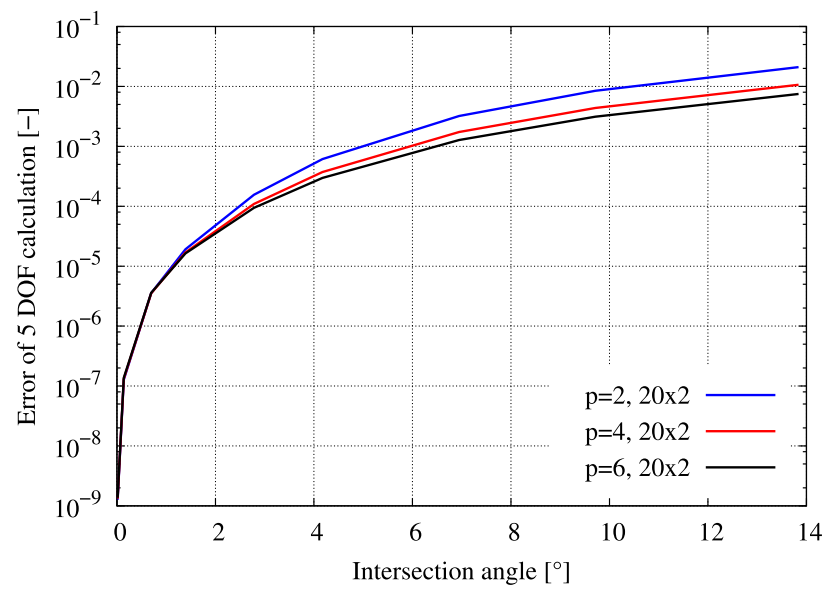

Figure 7: Simple shallow truss structure: Error of deformation at point $\mathbf{A}$ for calculations with five degrees of freedom at the intersection for $k$-refinement and $20 \times 2$ elements.

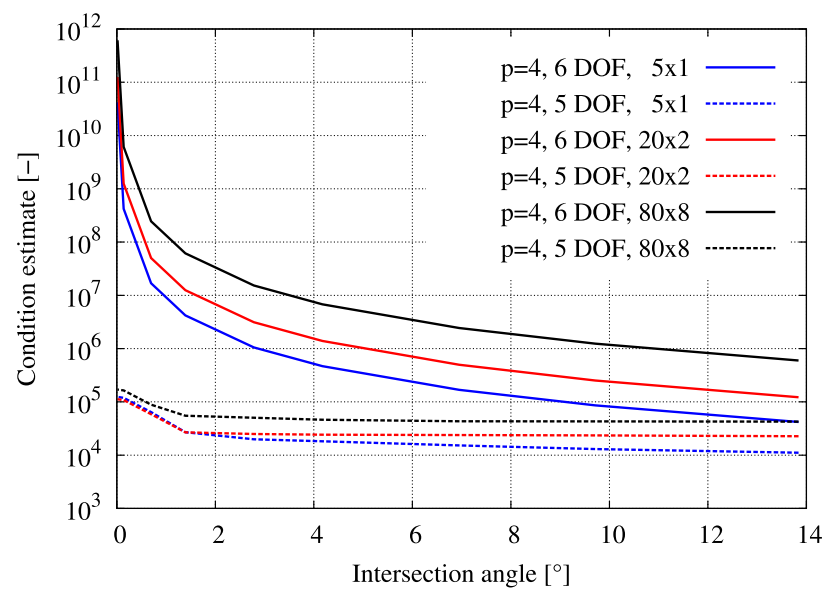

Figure 8: Simple shallow truss structure: Condition estimate for $h$-refinement with NURBS basis functions of order $p=4$. 


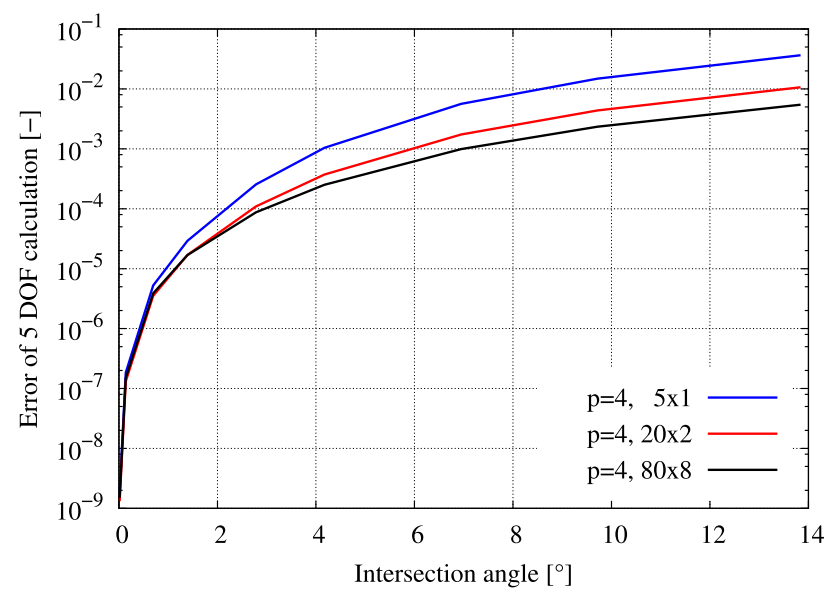

Figure 9: Simple shallow truss structure: Error of deformation at point $\mathbf{A}$ for calculations with five degrees of freedom at the intersection for $h$-refinement with NURBS basis functions of order $p=4$.

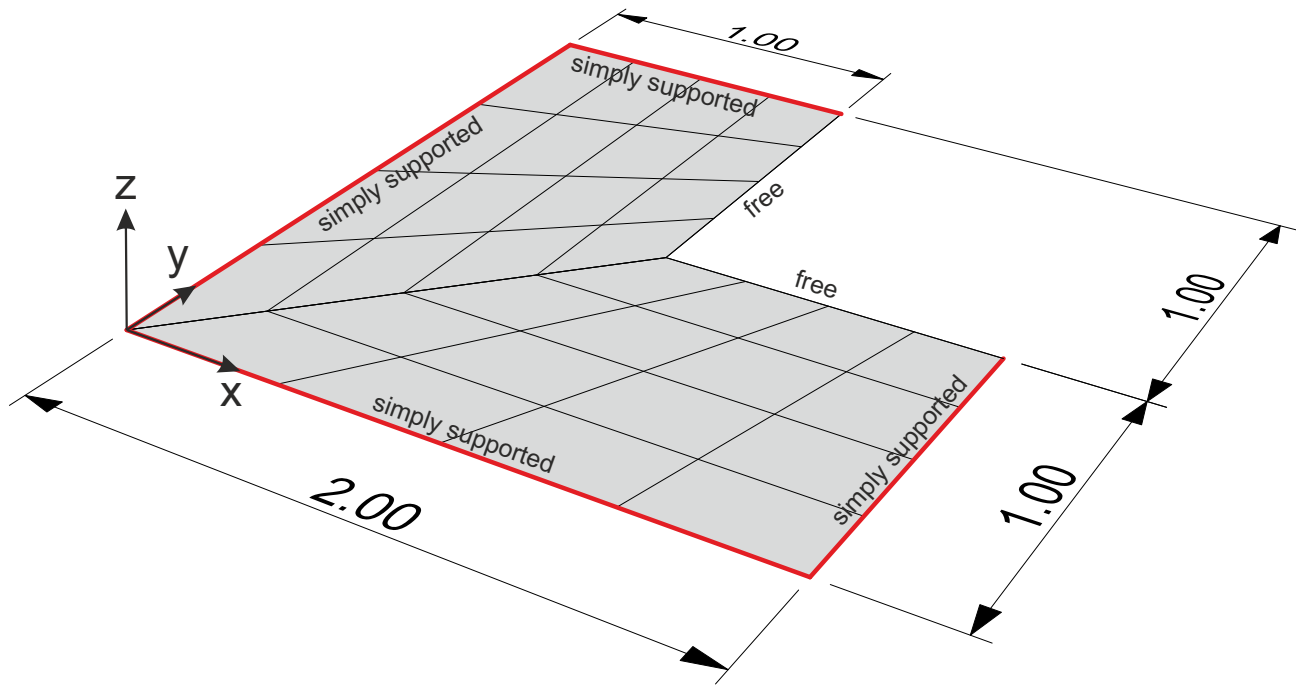

Figure 10: L-shaped plate: System sketch and coarsest mesh.

\subsection{Gravity-loaded L-shaped plate}

The concept of using two distinct sets of nodal basis system is depicted with the L-shaped plate loaded by gravity proposed in [9]. The geometry and the boundary condition are given in Fig. 10. The Young's modulus is $200 \cdot 10^{9}$ with a Poisson's ratio $v=0.3$. The shell thickness is $t=0.01$. The outer four edges are simply supported, while the two inward edges which intersect at the nook are free. An area load in negative $z$-direction of $p_{z}=784.532$ is applied, which is equal to the gravity load in [9]. The domain is modeled with two NURBS patches, which are $C^{0}-$ continuous along the shared edge. Thus, the first derivatives are discontinuous. Lamina basis systems computed from the first derivatives are used to determine the nodal basis systems. Thus, the nodal basis systems are not orthogonal with respect to the shared edge. The global nodal basis systems displayed in Fig. 11 provide unique rotational axes for all nodes. In Fig. 12 the patch wise nodal basis systems are plotted for both patches. The abrupt change in the first derivatives along the edge is reflected in differing nodal basis systems for the two control points associated to nodes along the connection line. The algorithm proposed in Sec. 6 automatically chooses two rotational degrees of freedom for the interface nodes. A convergence study for the deformation of the nook in $z$-direction is given in Fig. 13. The computations with NURBS basis function of order two, four and six are conducted with concept $b$ ) for 


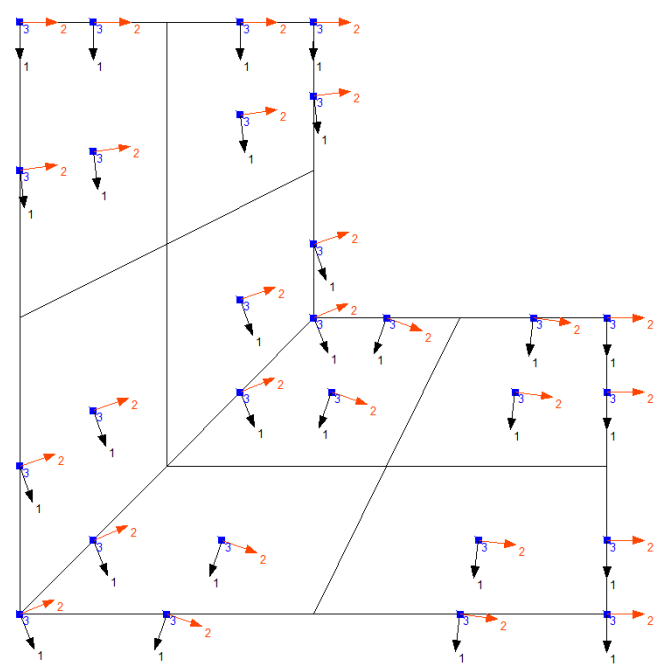

Figure 11: L-shaped plate: Global nodal basis systems.

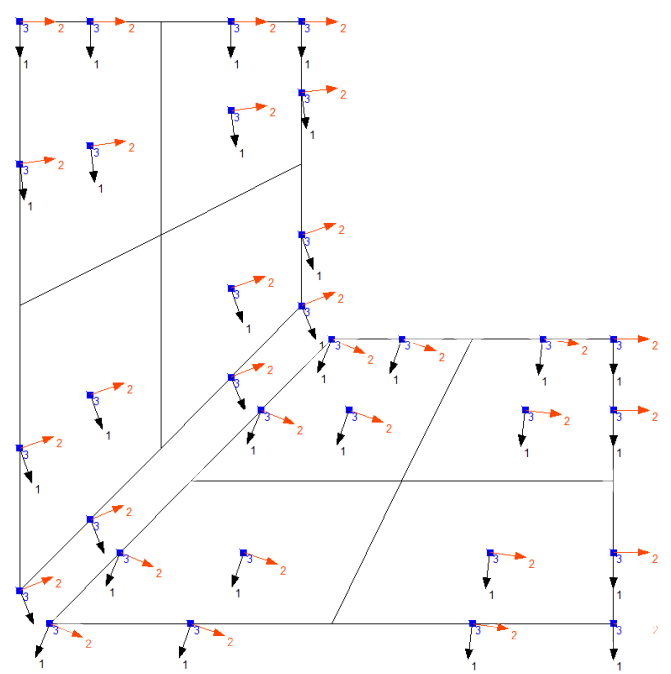

Figure 12: L-shaped plate: Patch wise nodal basis systems.

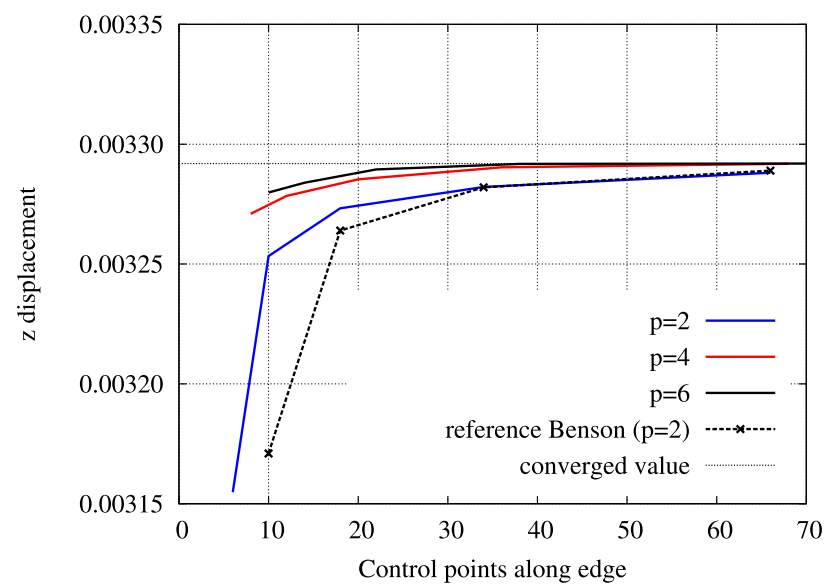

Figure 13: L-shaped plate: Convergence study for the $z$-displacement at the nook. 
the description of rotations. However, results attained with concept a) are almost not distinguishable as the domain is plane. Numerical Gauss integration is performed according to [28] with

$$
\operatorname{ceil}(p / 2)+1
$$

per parametric direction. The reference computations extracted from [9] are of order two and use the same number of integration points. The displacement $3.29195 \times 10^{-3}$ of a computation with $64 \times 64$ elements of $6^{\text {th }}$ order is used as converged value. The results in Fig. 13 show the excellent convergence behavior of the proposed formulation, especially for higher NURBS orders. This example shows that the proposed concept of two distinct sets of nodal basis systems abolishes the need for aligned parametrizations of neighboring patches at smooth interfaces. In standard Lagrange shell analysis with element wise nodal basis systems, the parametrizations have to be aligned, which is a severe restriction, see Sec. 7.2. To allow the computation of complex intersections, e.g. the pyramid in Sec. 8.4, usually drilling rotation stabilization has to be utilized.

\subsection{Channel section beam under point load}

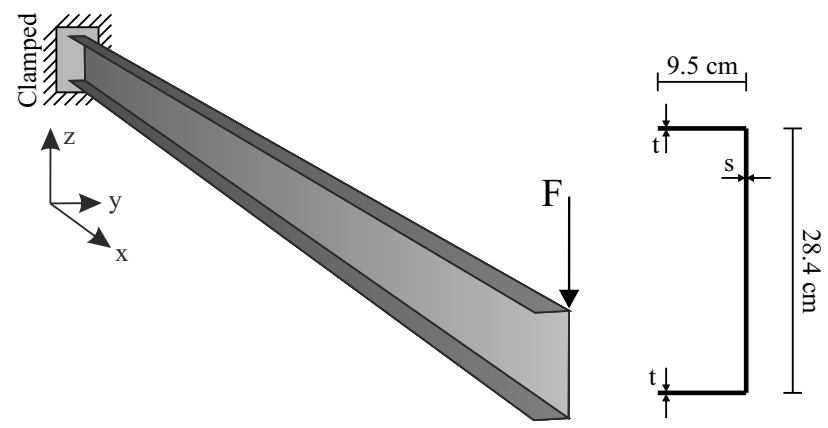

Figure 14: Channel section beam: System sketch.

The ability of the proposed formulation to handle multi-patch geometries with kinks is shown with the help of the channel section beam proposed in [26]. The cantilevered beam is clamped at one side and a point load $F$ is applied at the intersection of web and upper flange at the other side. A system sketch including the center line model of the cross-section is provided in Fig. 14. The length of the beam is $l=900 \mathrm{~cm}$. The Young's modulus is $E=21000 \mathrm{kN} / \mathrm{cm}^{2}$ and the Poisson's ratio is $v=0.3$. The wall thickness is $s=1.0 \mathrm{~cm}$ for the web and $t=1.6 \mathrm{~cm}$ for the flanges. The discretization of the coarsest mesh is chosen $18 \times 1$ for the flanges and $18 \times 3$ for the web. $h$-refinement is conducted by equal subdivision of all initial elements. The order is elevated using $k$-refinement. The control points on the intersection edges are automatically assigned three rotational degrees of freedom by the classification criteria introduced in Sec. 6. All other control points require only two rotational degrees of freedom. The convergence behavior is studied for linear as well as for nonlinear kinematics with large deformations and large rotations in the following. The reference Lagrange-based Reissner-Mindlin shell element formulation [29] is of order $p=1$ and describes rotations similar to concept a). However, due to the lack of geometric information, the nodes have to be classified manually. Computations with the isogeometric Reissner-Mindlin shell element of Benson et al. [11] implemented in the commercial code LS-DYNA serve as a further reference. A current development version from January 2014 was used for the calculations. Here the classification of nodes is avoided by using three global rotations for every control point in combination with drilling rotation stabilization.

In the linear case a force $F=1 \mathrm{kN}$ is applied. In Fig. 15 the vertical deformations $\Delta z$ of the loaded control point are plotted. They are normalized to $\Delta z=-1.483700 \mathrm{~cm}$. This result is attained with the proposed shell formulation using $360 \times 100$ elements of order $p=6$ and is used as a converged result in the following. The results of the present formulation do not differ perceivably between the two concepts for the description of rotations, as all patches are plane. Thus, only one curve is plotted for every order of the present formulation. In Fig. 16 the error with respect to the converged value is given in a double-logarithmic diagram to show the behavior for fine meshes. The results clearly show proper convergence behavior of the proposed formulation for $h$-refinement and for order elevation. The 


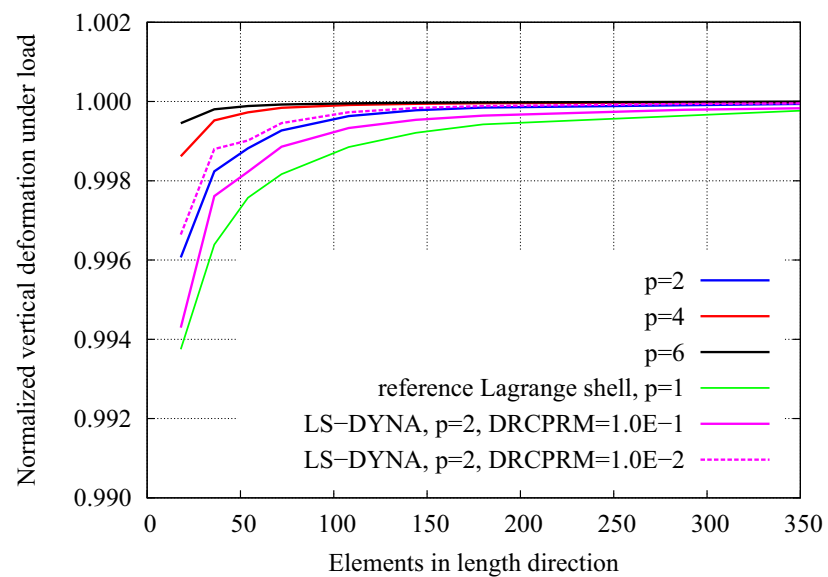

Figure 15: Channel section beam: Normalized deformation for linear computations with $F=1 \mathrm{kN}$.

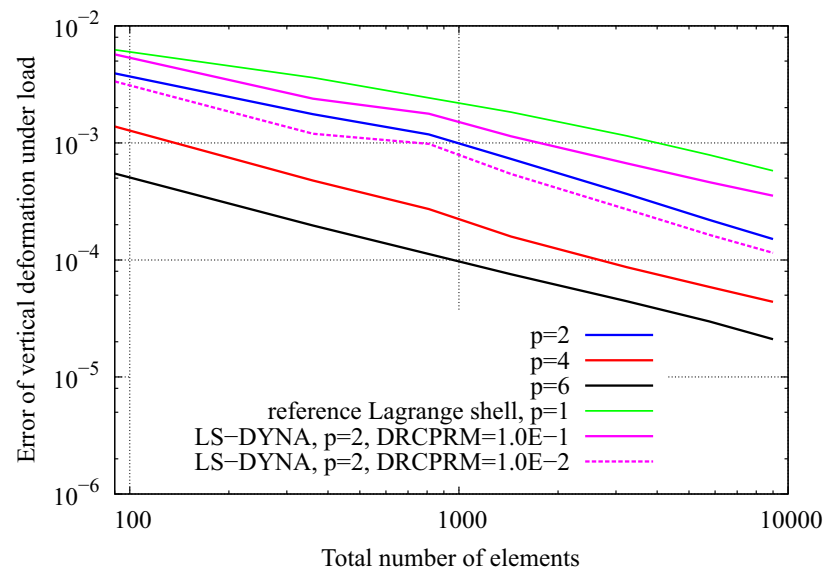

Figure 16: Channel section beam: Error of deformation for linear computations with $F=1 \mathrm{kN}$ (legend see Fig. 15).

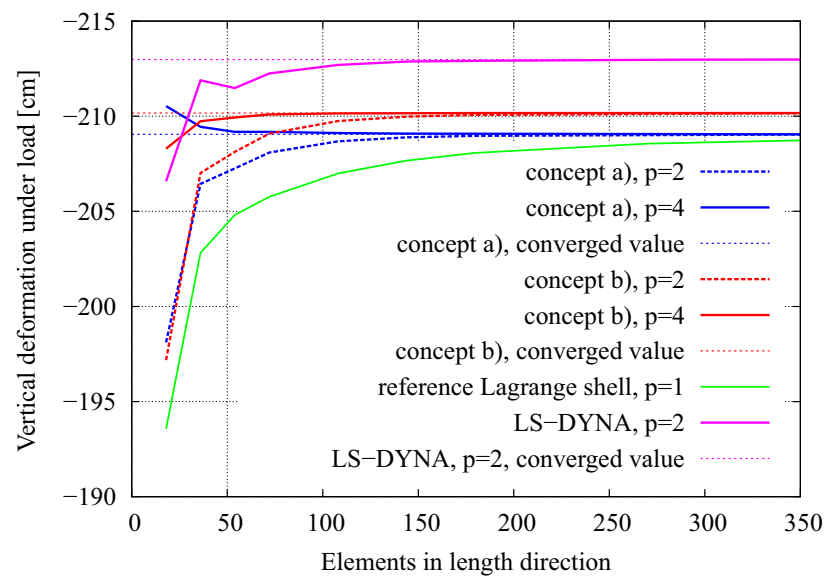

Figure 17: Channel section beam: Deformation for nonlinear computations with $F=15 \mathrm{kN}$. 


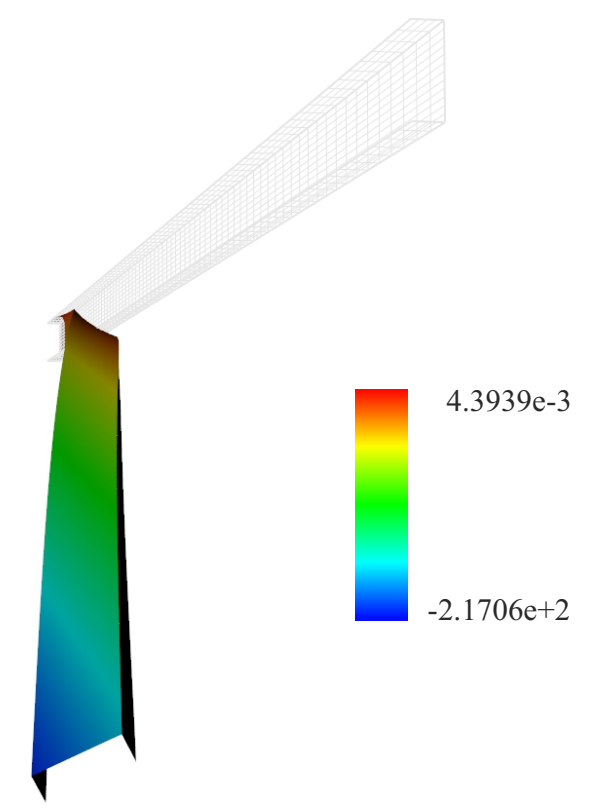

Figure 18: Channel section beam: Initial and deformed geometry for a load $F=15 \mathrm{kN}$ with $z$-displacement as isosurface computed with $72 \times 20$ elements of $2^{\text {nd }}$ order using concept $b$ ).

reference computations with the Lagrange-based shell formulation converge to the same value. For the reference isogeometric Reissner-Mindlin shell in LS-DYNA two curves are plotted, as the deformation convergence behavior slightly depends on the choice of the drilling rotation stabilization parameter DRCPRM. The converged deformation has the same value as for the proposed shell formulation. It has to be noted, that all formulations yield very good results ( $\geq 99.4 \%$ of the converged deformation) even for the coarsest mesh.

In the nonlinear case a force $F=15 \mathrm{kN}$ is applied in 30 uniform load steps. The vertical deformations are plotted in Fig. 17. Here the converged values for $360 \times 100$ elements of $4^{\text {th }}$ order differ between the two proposed concepts for the description of the rotations. Concept a) results in a slightly smaller deformation $\Delta z=-209.05 \mathrm{~cm}$ in comparison to $\Delta z=-210.16 \mathrm{~cm}$ for concept $b$ ). In Fig. 18 a plot of the initial and the deformed system illustrates the magnitude of the deformations and rotations. The reference Lagrange shell converges naturally against the same value as the proposed isogeometric shell with concept $a$ ). The computations in LS-DYNA were performed with the preset value for the drilling rotation stabilization parameter (DRCPRM=1). The converged deformation $\Delta z=-212.98 \mathrm{~cm}$ under the load is slightly larger than the converged deformations computed with the proposed shell formulations. It is computed with $360 \times 100$ elements of $2^{\text {nd }}$ order and with reduced integration (INT=0). The differences between the three converged displacements - concept a) yields $0.53 \%$ smaller and LS-DYNA yields $1.34 \%$ larger deformations than concept $b$ ) - are very small taken into account that the problem is highly nonlinear and features large displacements and rotations. An explanation for the difference in converged displacements between both proposed concepts can be found in Sec. 7.4.

\subsection{Pyramid under surface load}

Complex intersections of multiple patches - both smooth and with kinks - are tested with the proposed pyramid. A sketch of the geometry is given in Fig. 19. All necessary dimensions to construct the nine patches of the pyramid are provided in Fig. 20. The three faces are modeled by three patches each to avoid the usage of degenerated patches. The base of the pyramid is fully clamped. The height of the pyramid is 5.0 with a wall thickness $t=0.1$. A Young's modulus $E=2.1 \cdot 10^{4}$ and a Poisson's ratio $v=0.3$ are used. The pale shaded front face of the pyramid is loaded with an outward normal surface load $n=1.0$, whereas the two darker shaded back faces are loaded with an inward normal surface load $n=-1.0$. The coarsest mesh uses one element per patch. These nine initial elements are subdivided in both parametric directions with the subdivision factor used in Fig. 21 as abscissa. The finest mesh 


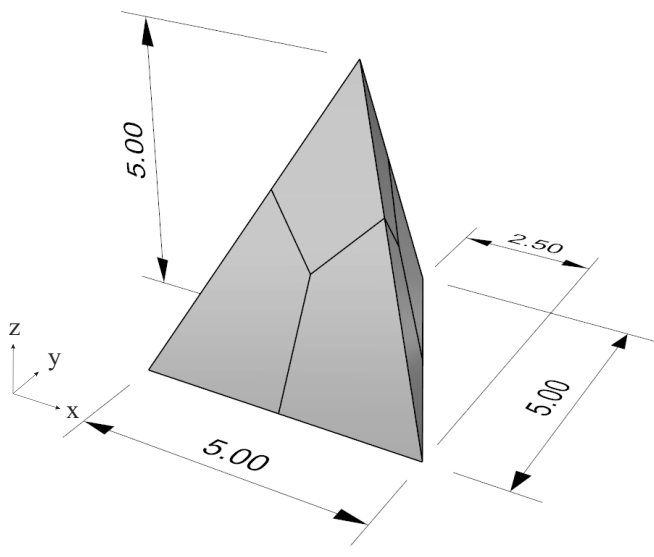

Figure 19: Pyramid: System sketch with coarsest mesh.

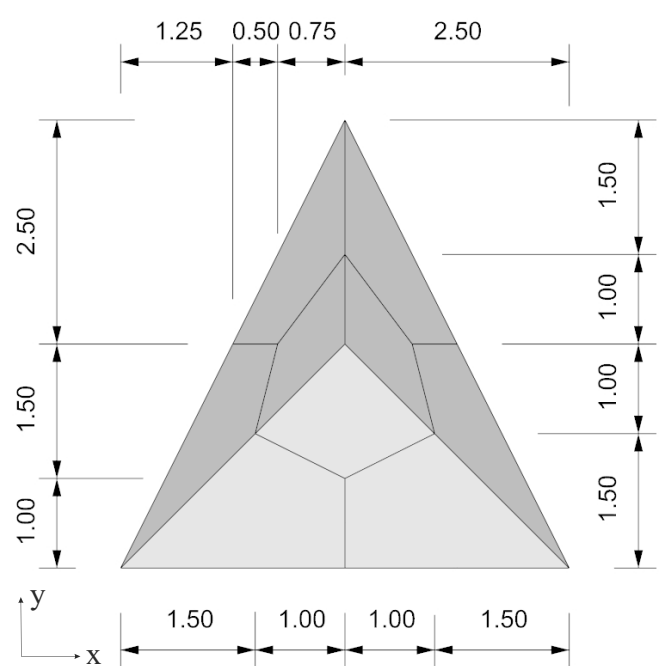

Figure 20: Pyramid: top view of the geometry modeled with nine patches.

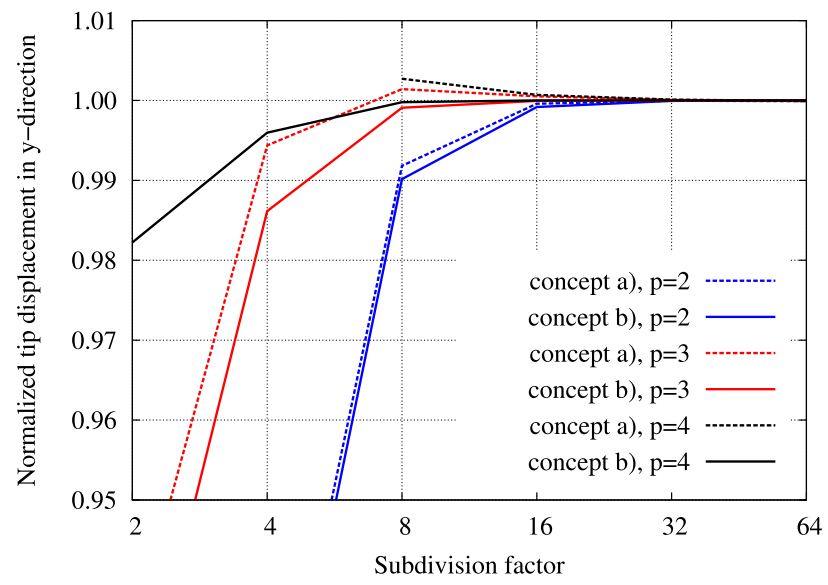

Figure 21: Pyramid: Tip displacement for nonlinear computations. 
computed thus consists of nine patches with $64 \times 64$ elements each, summing up to 36864 elements. The order of the NURBS basis functions is elevated using $k$-refinement. The control points located on the patch intersections with kinks are automatically assigned six degrees of freedom. All other control points, including those at the smooth patch intersection within the faces, use only five degrees of freedom, as no stiffness against drilling rotations arises there. The computation of this geometry with conventional shell elements requires the usage of drilling rotation stabilization, see Sec. 7.2.

Linear computations of the problem setup showed - in agreement to the results of Sec. 8.3 - no perceivable differences between computations with concept $a$ ) and concept $b$ ). The tip displacement of geometrically nonlinear computations is plotted in Fig. 21. The displacement $u_{y}=-0.0301409$, obtained with 36864 elements of $4^{\text {th }}$ order using concept $b$ ), is used for a normalization of the displacements. For the same mesh concept a) yields slightly smaller deformations $u_{y}=-0.0301390$. The computations with concept $b$ ) show good convergence behavior for $h$-refinement, as well as for $k$-refinement. This is not entirely true for concept $a$ ). Computations with order $p=2$ and $p=3$ show comparable and good behavior. But computations with order $p=4$ need much more elements to reach the same level of accuracy than with concept $b$ ). For a subdivision factor of 2 and 4 no equilibrium state could be found with concept a). With rising order of NURBS the stability and the accuracy of concept a) decreases. The current configuration is curved and thus, the results differ between both concepts, with concept $b$ ) yielding superior results. This behavior is in accordance to the numerical examples published by the authors in [6]. Nevertheless, computations with concept a) using low orders of NURBS are competitive as the formation of the stiffness matrix is less expensive.

\subsection{Partly clamped hyperbolic paraboloid with stiffeners}

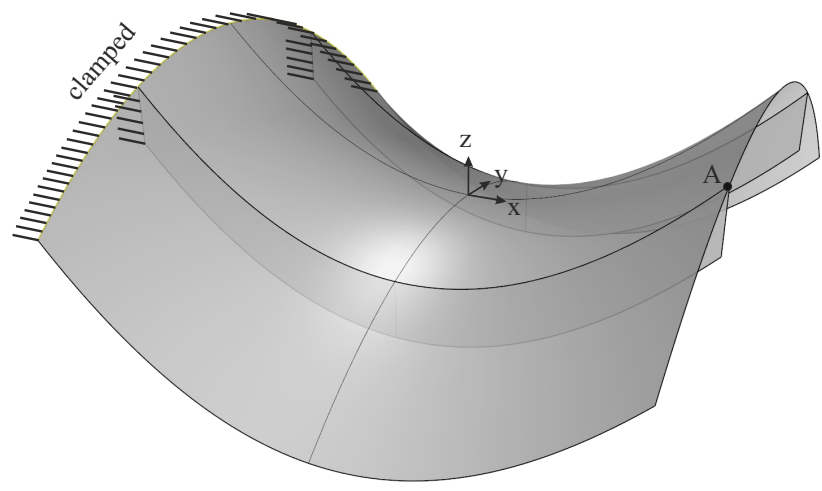

Figure 22: Hyperbolic paraboloid: System sketch with coarsest mesh.

The hyperbolic paraboloid described by NURBS was firstly used in [14]. With additional stiffeners an example is created, which combines curved geometries and kinks. The geometry is simple and easy to construct. The main surface is described by the function

$$
z=x^{2}-y^{2} \quad(x, y) \in[-0.5 ; 0.5]
$$

with a shell thickness $t=0.04$. The two stiffeners are described by

$$
y= \pm 0.25 \quad z=x^{2}-0.0625-0.1 u \quad x \in[-0.5 ; 0.5]
$$

with the surface parameter $u \in[0 ; 1]$. The thickness of the stiffeners is chosen $s=0.01$. A Young's modulus $E=$ $2.1 \cdot 10^{4}$ and a Poisson's ratio $v=0.3$ are used. The surface is loaded with an area load in $z$-direction $q_{z}=-0.9$. Both the surface and the stiffener are clamped at $x=-0.5$. A sketch of the system including the coarsest mesh is provided in Fig. 22. The twelve initial elements are subdivided in both parametric directions with the subdivision factor used in Fig. 24 as abscissa. The distinction criterion automatically determines the nodes requiring three rotational degrees of freedom, although those nodes are not located on the kink, as can be seen in Fig 23. Computations are performed with NURBS of order $p=2$ and $p=4$, where the order is elevated by $k$-refinement. The initial symmetric geometry is completely discretized to allow the occurrence of asymmetric equilibrium states. 


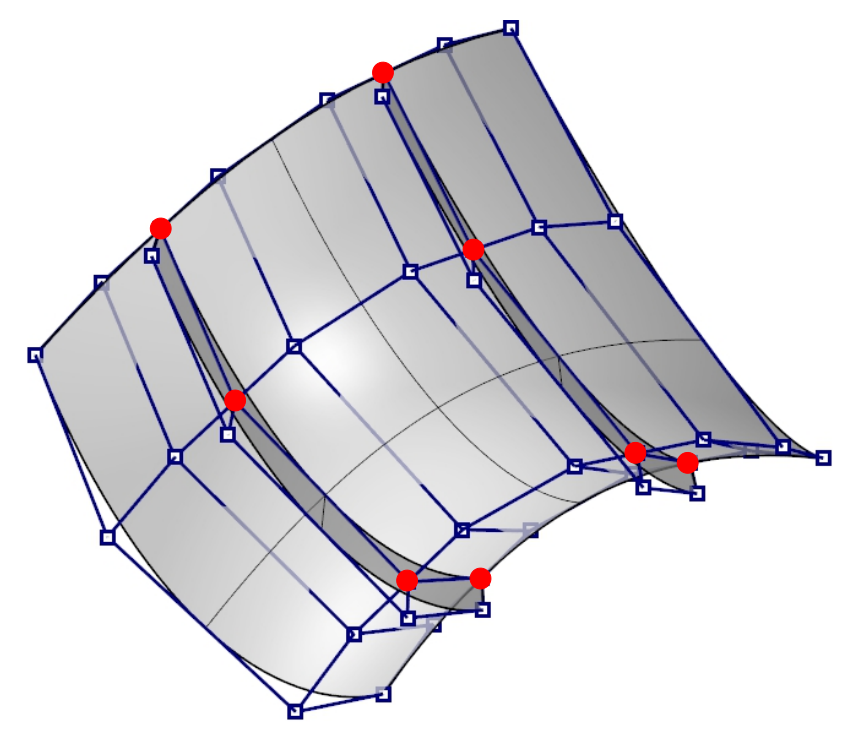

Figure 23: Hyperbolic paraboloid: Initial mesh with control points displayed. Nodes requiring three rotational degrees of freedom are marked with a red filled circle.

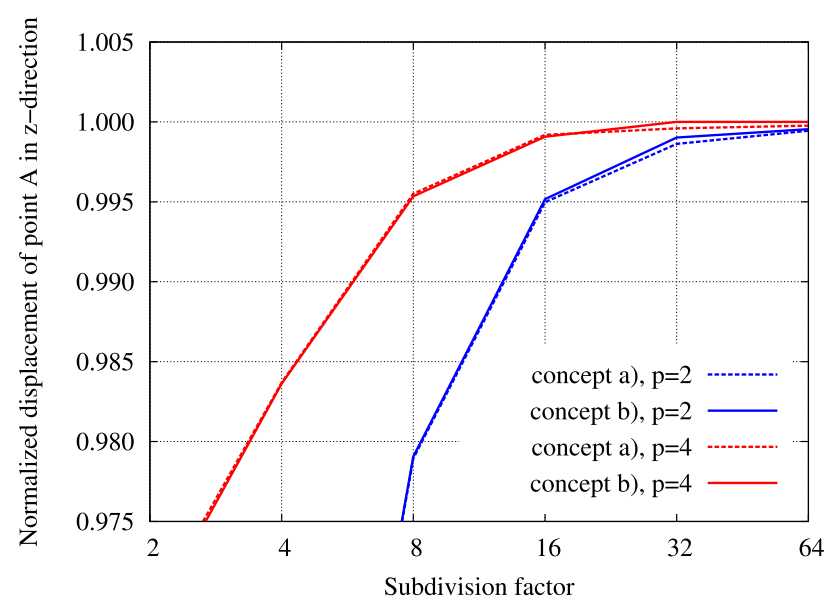

Figure 24: Hyperbolic paraboloid: Deflection of point A in z-direction.

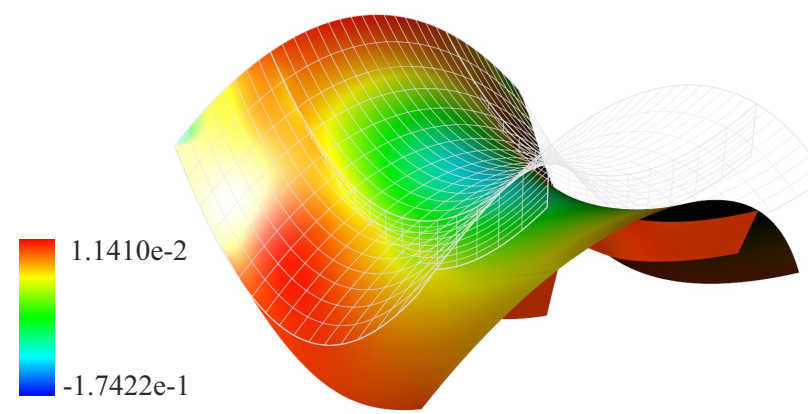

Figure 25: Hyperbolic paraboloid: Undeformed mesh (not all elements displayed for clarity) and deformed mesh with bending moment $m_{22}$ as isosurface for $p=4$ with a refinement factor 64 using concept $b$ ). 
The applied load yields large deformations and large rotations. This is illustrated in Fig. 25, where the initial mesh is given in comparison to the deformed mesh without elevation. In addition the moment $m_{22}$ is plotted as isosurface onto the deformed mesh. The rather thin stiffeners buckle locally under the applied load, which impairs the equilibrium iteration. Nevertheless, almost all computed discretizations are able to compute the system in one load step. The deflection of point A in z-direction is plotted in Fig. 24. The results of both concepts are very similar and show very good convergence behavior for $h$ - and for $k$-refinement. For a refinement factor 64 and order $p=4$ concept a) results in a slightly smaller deformation than concept $b)(-0.198602$ vs. -0.198649$)$. The number of iterations needed is always in the same range with no concept being superior to the other. The deformation converge behavior of this problem setup is seriously influenced by the formation of local buckles. This fact is illustrated in Fig. 26 with plots of the deformed structure for four meshes of $4^{\text {th }}$ order computed with concept $a$ ). The deformations are superelevated by factor 2 and the deformations in $y$-direction (normal with respect to the initial configuration of the stiffeners) are plotted as isosurface. For the first mesh, with a refinement factor 2, the deformation plot given in Fig. 26a reveals

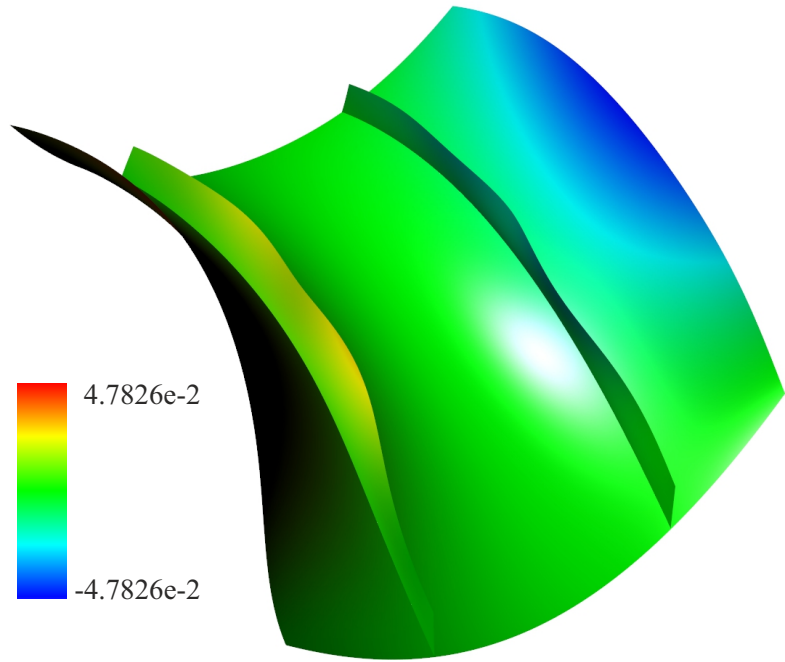

(a) $p=4$, refinement factor 2

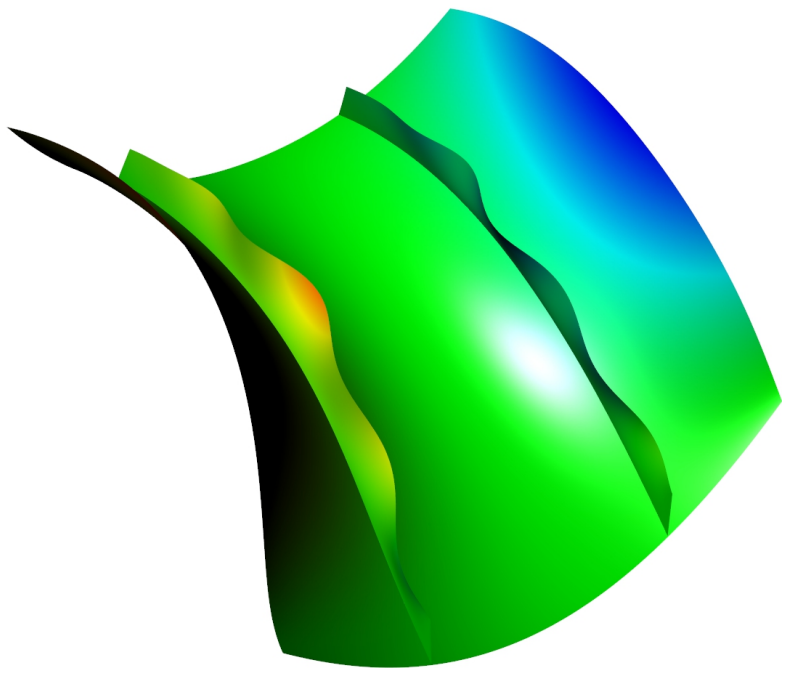

(c) $p=4$, refinement factor 8

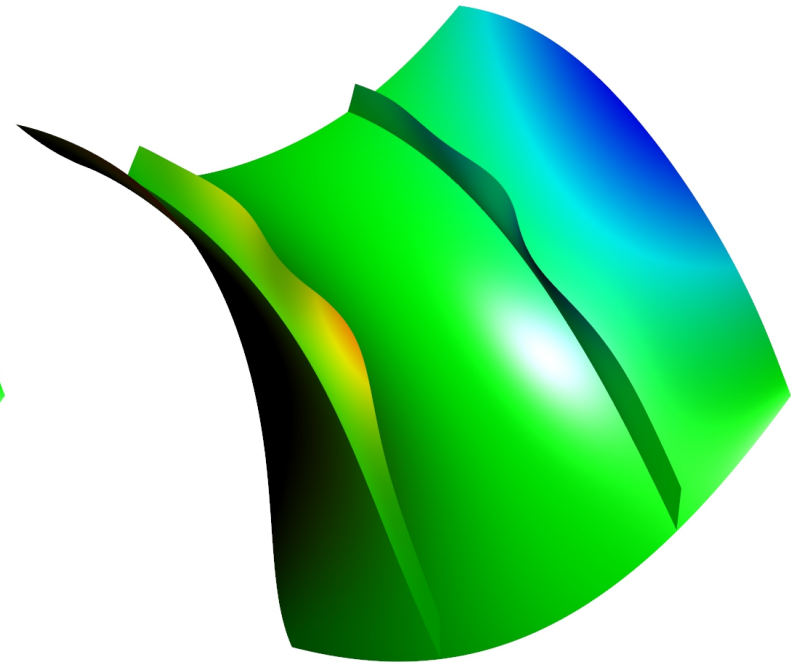

(b) $p=4$, refinement factor 4

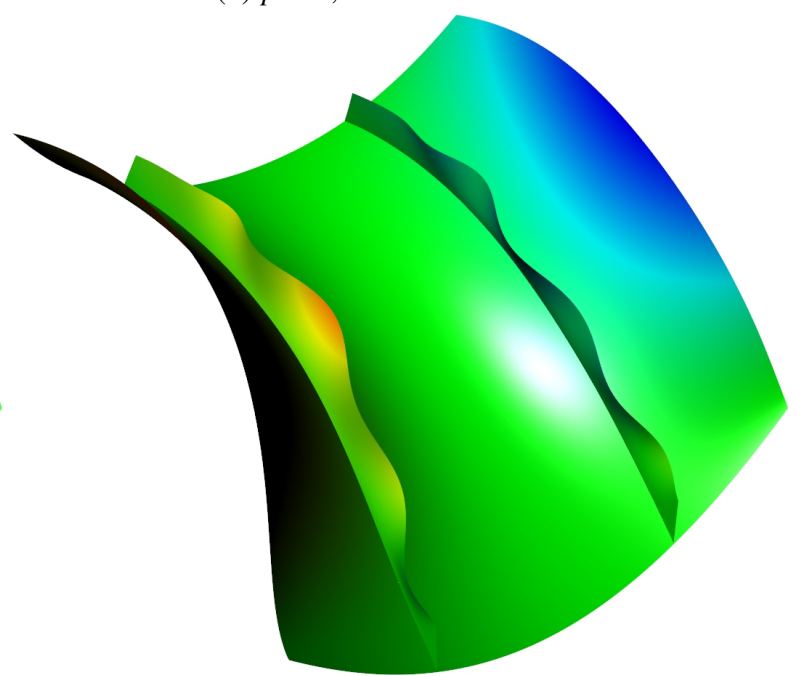

(d) $p=4$, refinement factor 64

Figure 26: Hyperbolic paraboloid: Plot of deformed structure with superelevation factor 2. Colored isosurfaces represent $y$-deformation (view from below, lower right side is the clamped side). 
two inward buckles. The deformation plot for a refinement factor 4 in Fig. 26 b shows still two buckles with larger amplitude. The next subdivision step given in Fig. 26c yields a jump into the next eigenform, having one outward buckle at the clamped side and three subsequent inward buckles. Computations with a subdivision factor 16 show the same buckles, but with larger amplitudes. The plots for subdivision factors 16, 32 and 64 are not distinguishable from each other by the eye. Thus, only the finest one is provided in Fig. 26d. The difference between the two concepts is very low for this numerical example, which has constant curvature.

\subsection{Free form surface with arbitrary curvature and kink}

All numerical examples presented above are flat or have a rather small and constant curvature in the reference configuration. Thus, a free form surface with strongly changing curvature and a kink is proposed. It consists of two patches that are smooth within but joined with a kink. The angle of the kink is not constant but changes from $90^{\circ}$ to almost $0^{\circ}$ along the joint. This example allows a comparison of the deformation behavior between both proposed concepts for arbitrary curvatures. Furthermore, the influence of the limit intersection angle $c_{\text {angle }}$ can be studied due to the angle of the kink approaching $0^{\circ}$. The example is an extension of the free form surface proposed in [6], which was designed to test the proper treatment of rotations. The data needed to construct the geometry is given in Appendix A. The lower edge $(z=0)$ is fully clamped, all other edges are free. The upper edge of patch 1 is loaded with a line load $p_{y}=10$. The wall thickness is $t=0.1$, the Young's modulus is $E=1.2 \cdot 10^{6}$ and the Poisson ratio is $v=0.3$. A system sketch is provided in Fig. 27. For NURBS basis functions with orders $p=3$ to $p=5 h$-refinement studies are performed. All elements of the coarsest mesh displayed in Fig. 27 are subdivided in both parametric directions. The subdivision factor is used as abscissa in Figs. 28 and 29.

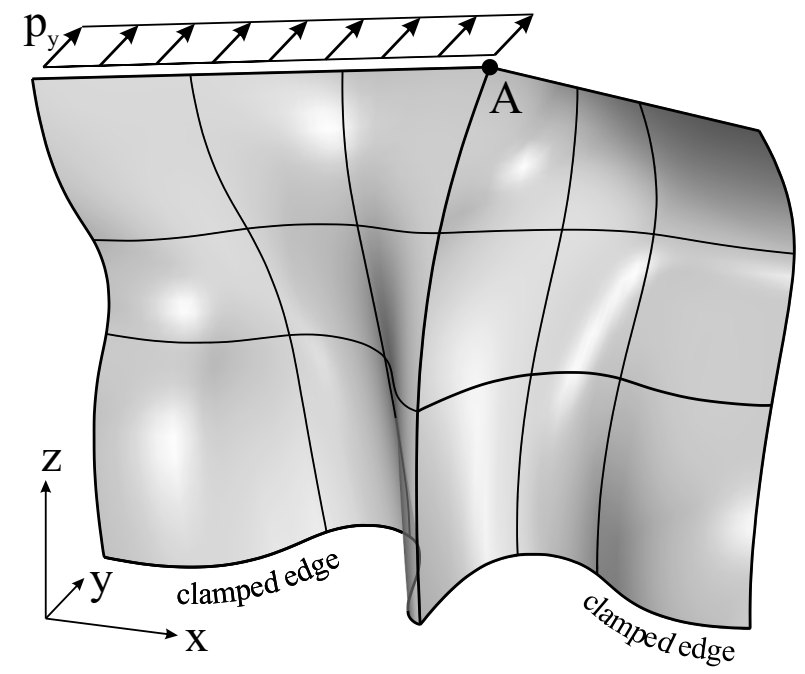

Figure 27: Free form surface with arbitrary curvature and kink: System sketch and coarsest mesh.

The deformation results for linear computations are given in Fig. 28. They are normalized to a value $\Delta y_{A}=$ 0.60002. This value is computed with a subdivision factor 64, which results in 73728 elements. The order of the basis functions is $p=4$ and concept $b$ ) is used. Both concepts converge against the same deformation. For an order $p=3$ the deformations computed with both concpets are very similar. Concept $a$ ) entails slightly better results than concept $b$ ). For orders of $p=4$ and $p=5$ the situation is different. Here concept $a$ ) obviously overestimates the deformations for subdivision factors of 8 and 16, whereas the deformations of concept $b$ ) are not distinguishable by the eye from the converged solution. The results of concept a) deteriorate with rising order of the NURBS basis functions. This behavior is in accordance to the findings of [6]. All computations plotted in Fig. 28 are performed with a limit intersection angle $c_{\text {angle }}=2^{\circ}$. The angle of the kink goes below $2^{\circ}$ along the common edge. Thus, for fine meshes, depending on the value of $c_{\text {angle }}$, a small number of control points on the kinks is classified wrongly as smooth nodes. Tab. 1 provides deformation results for different values of $c_{\text {angle }}$ for a subdivision factor 64 and order $p=4$. For the smallest value all control points along the kink are classified correctly as nodes on kink. The deviation 
of results for $c_{\text {angle }}=2^{\circ}$ from those for $c_{\text {angle }}=0.05^{\circ}$ is in the range of $10^{-5}$ for both concepts. Thus, in the linear case the choice of $c_{\text {angle }}$ has negligible influence.

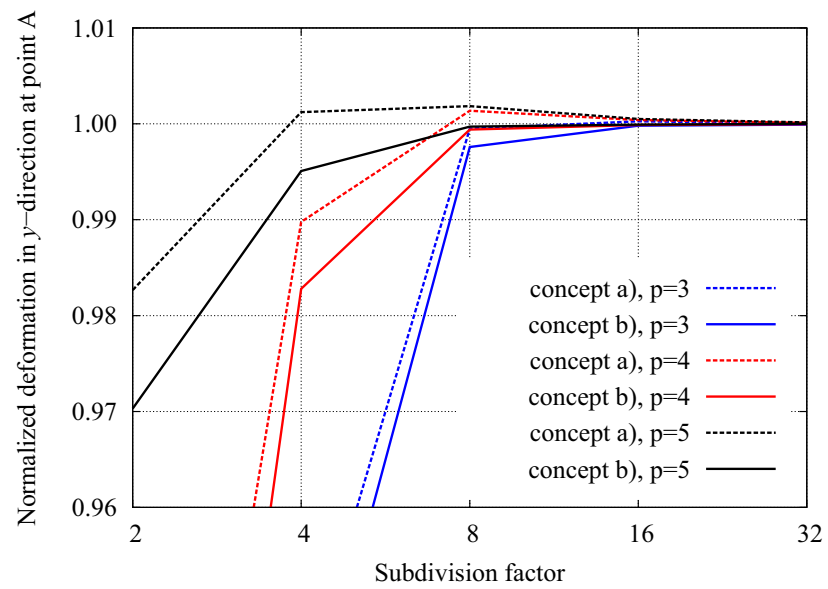

Figure 28: Free form surface with arbitrary curvature and kink: Deformation for linear computations.

\begin{tabular}{|c|c|c|c|}
\hline \multirow[t]{2}{*}{$c_{\text {angle }}$} & \multirow{2}{*}{$\begin{array}{l}\text { smooth nodes } \\
\text { on the kink }\end{array}$} & \multicolumn{2}{|c|}{$y$-deformation of point $\mathrm{A}$} \\
\hline & & concept a) & concept b) \\
\hline $2^{\circ}$ & 3 & 0.60004559 & 0.60002578 \\
\hline $0.2^{\circ}$ & 1 & 0.60003666 & 0.60001975 \\
\hline $0.05^{\circ}$ & 0 & 0.60003562 & 0.60001974 \\
\hline
\end{tabular}

Table 1: Free form surface with arbitrary curvature and kink: Influence of the limit intersection angle for linear computations for a subdivision factor 64 and order $p=4$.

A nonlinear convergence study is given in Fig. 29. The same load as in the linear case is applied in one load step. The deformation convergence behavior of concept $b$ ) is, akin to the linear case, very good. All computations show good convergence in the Newton-Raphson equilibrium iteration with a limit intersection angle $c_{\text {angle }}=2^{\circ}$, and in each case 11 iterations are sufficient. The choice of $c_{\text {angle }}$ has only minor influence for concept $b$ ), see the right column of Tab. 2. The numerical stability is not affected, even for very small values of $c_{\text {angle. }}$. The results for concept a) converge against a slightly larger value for the deformation of point A. See Sec. 7.4 for an explanation. Computations with order $p=3$ show slightly inferior deformation convergence behavior, but here also 11 iterations in the Newton-Raphson iteration suffice, and $c_{\text {angle }}=2^{\circ}$ can be used. Computations with concept $a$ ) and higher orders reveal a different picture. For an order $p=4$ computations with a subdivision factor 4 do not converge. For a subdivision factor of 64 the limit intersection angle has to be increased to prevent numerical instabilities. A value $c_{\text {angle }}=10^{\circ}$ has been used. Computations with concept $a$ ) and order $p=5$ do not converge at all in one load step for $c_{\text {angle }}=2^{\circ}$. With two load steps and $c_{\text {angle }}=10^{\circ}$ equilibrium could be established, but due to the incorrect treatment of control points on the kink as smooth the resulting deformations largely deviate from the results for $3^{\text {rd }}$ and $4^{\text {th }}$ order. Thus, the results are not displayed in Fig. 29. For concept $a$ ) the choice of the limit intersection angle $c_{\text {angle }}$ has significant influence on the stability of the Newton-Raphson iteration, see Tab. 2. For a subdivision factor 32 and $4^{\text {th }}$ order basis functions divergence occurred for $c_{\text {angle }}=0.5^{\circ}$.

For this example concept $b$ ) is less sensitive to small values of $c_{\text {angle }}$ than concept $a$ ). The reason for this difference is as follows. In concept a) stiffness against drilling rotations arises only due to the intersection of the patches at the kink. Thus for a very small intersection angle almost no stiffness against drilling rotations arises, which entails a bad condition of the stiffness matrix. In concept $b$ ) stiffness against drilling rotations also arises due to the curvature of the elements, which adjoin the kink. If the vector $\mathbf{a}_{3}^{h}$ of the interpolated basis system in the integration point deviates from the nodal basis vector $\widetilde{\mathbf{a}}_{3 I}$ of the node on the kink, than the matrix $\mathbf{M}_{I}$ defined in (71) ensures numerical stability also for intersection angles approaching zero. This example clearly shows that for geometries with larger and changing 
curvature concept $b$ ) is superior to concept $a$ ) in terms of robustness and accuracy.

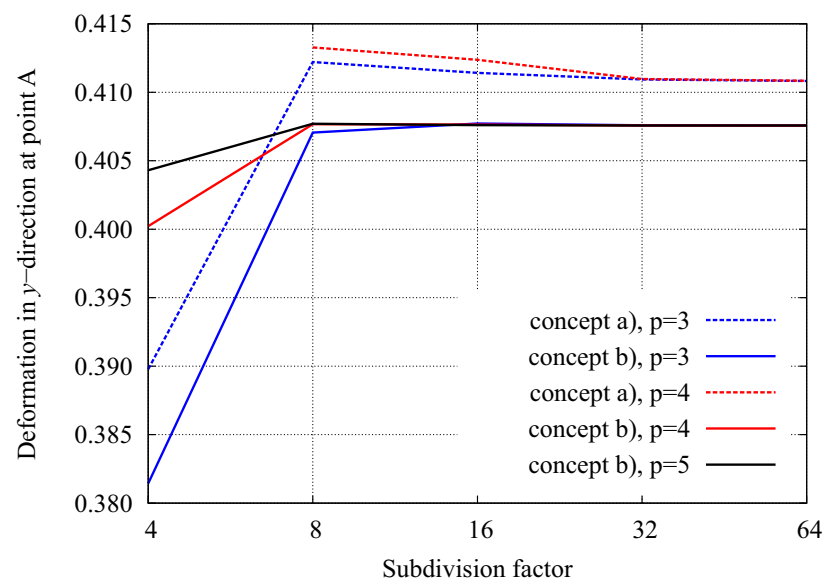

Figure 29: Free form surface with arbitrary curvature and kink: Deformation for nonlinear computations.

\begin{tabular}{c|c|c|c}
$c_{\text {angle }}$ & smooth nodes & \multicolumn{2}{|c}{$y$-deformation of point A } \\
& on the kink & concept a) & concept $b$ ) \\
\hline $2^{\circ}$ & 2 & 0.41096483 & 0.40756868 \\
$1^{\circ}$ & 1 & 0.41094670 & 0.40756555 \\
$0.5^{\circ}$ & 0 & divergence & 0.40756441
\end{tabular}

Table 2: Free form surface with arbitrary curvature and kink: Influence of the limit intersection angle for nonlinear computations for a subdivision factor 32 and order $p=4$.

\section{Conclusion}

A framework for the automated computation of complex geometries with an isogeometric Reissner-Mindlin shell has been presented. In demanding numerical examples the high accuracy and robustness of the shell formulation for large deformations and rotations has been shown. Both presented concepts for the description of rotations showed comparable results for the presented examples. Prior work of the authors [6] asserted, that the accuracy of computations of geometries with changing or strong curvature highly benefits from employing concept $b$ ), while for plane or lightly curved geometries concept $a$ ) suffices. These findings also hold true for geometries with kinks and intersections. Furthermore, the easy applicability of the complete framework has been shown. The usage of drilling rotation stabilization can completely be avoided by the combination of all proposed methods. The distinction criterion proposed in Sec. 6 requires an empirical threshold parameter, which has to be chosen by the user at this time. Future work might find a generally valid value to avoid user interaction. The decisive ingredients for the complete framework are:

- A method for the computation of nodal basis systems which are able to interpolate the director vector exactly even in the proximity of kinks. The nodal basis systems also serve as unique rotational axes for the nodal rotational degrees of freedom.

- A distinction criterion for an automatic classification of nodes into nodes on kinks and nodes in smooth regions. Accordingly, the number of rotational degrees of freedom is assigned to ensure on the one side a proper transmission of rotations at kinks and at the other side a proper condition of the stiffness matrix.

- A Reissner-Mindlin shell formulation which is able to handle complex geometries including kinks and allows the intersection of patches with arbitrary parametrization. Both provided concepts for the description of rotations fulfill these criteria. 
These facts allow finite element programs based on the proposed framework to make full use of the advantages of NURBS-based isogeometric analysis. The existing information of the exact geometry can be used inside the numerical simulation kernel to attain more realistic results. Especially in large deformation and stability analysis the omission of drilling rotation stabilization renders results more precise and reliable.

Future work might be concerned with enriching the proposed formulation with methods to compute non-conforming meshes and trimmed NURBS surfaces. Suitable methods for these objectives are presented in [30], respectively [31]. With these additional ingredients arbitrary real-world NURBS models could be computed without alteration of the mesh.

\section{Acknowledgement}

The authors greatly acknowledge the fruitful discussions and cooperation with Prof. Kai-Uwe Bletzinger, Dr. Roland Wüchner and Michael Breitenberger from the Chair of Structural Analysis at Technische Universität München. The authors thank for the dissemination of parts of their design-through-analysis tool chain. Furthermore, thank is due to Dr. Stefan Hartmann from Dynamore $\mathrm{GmbH}$ and an anonymous referee for their support regarding the calculations with LS-DYNA.

\section{Appendix A. Geometry of the free form surface with arbitrary curvature and kink}

The free form surface with arbitrary curvature and kink used in Section 8.6 consists of two patches with changing curvature. Both patches are Gordon-Coons surfaces. Thus, each patch is constructed uniquely from its four boundary B-Spline curves. In Tab. A.1 the control points for all boundary curves are given. As $w=1$ holds for all control points B-Spline surfaces are formed. The knot vector

$$
\boldsymbol{\Xi}=\left[0,0,0,0, \frac{1}{3}, \frac{2}{3}, 1,1,1,1\right]
$$

is identical for all curves. The order of the B-Spline basis functions is $p=3$ and the number of control points is $n=6$. Thus, the coarsest mesh consists of $3 \times 3$ elements for each patch.

\begin{tabular}{l|c|c|c|c} 
Patch 1 & top & bottom & left & right \\
\hline Control points & $0,0,15$ & $0,0,0$ & $0,0,0$ & $11,0,0$ \\
$(\mathrm{x}, \mathrm{y}, \mathrm{z})$ & $\frac{11}{9}, \frac{2}{3}, 15$ & $5,0,0$ & $0,0,5$ & $11,0, \frac{8}{3}$ \\
& $\frac{11}{3}, 2,15$ & $5,5,0$ & $0,2,7$ & $11, \frac{2}{9}, \frac{62}{9}$ \\
& $\frac{22}{3}, 4,15$ & $10,5,0$ & $0,2,10$ & $11, \frac{17}{9}, \frac{101}{9}$ \\
& $\frac{88}{9}, \frac{16}{3}, 15$ & $10,0,0$ & $0,0,12$ & $11, \frac{13}{3}, \frac{41}{3}$ \\
& $11,6,15$ & $11,0,0$ & $0,0,15$ & $11,6,15$ \\
\hline Patch 2 & top & bottom & left & right \\
\hline Control points & $11,6,15$ & $11,0,0$ & $11,0,0$ & $20,3,0$ \\
(x,y,z) & $12.8,4.8,15$ & $11,3,0$ & $11,0, \frac{8}{3}$ & $20,2,3$ \\
& $14.6,3.6,15$ & $12,5,0$ & $11, \frac{2}{9}, \frac{62}{9}$ & $20,4,7$ \\
& $16.4,2.4,15$ & $15,5,0$ & $11, \frac{17}{9}, \frac{101}{9}$ & $20,8,10$ \\
& $18.2,1.2,15$ & $17,2,0$ & $11, \frac{13}{3}, \frac{41}{3}$ & $20,4,13$ \\
& $20,0,15$ & $20,3,0$ & $11,6,15$ & $20,0,15$
\end{tabular}

Table A.1: Control points of the boundary curves.

\section{References}

[1] M. Bischoff, W. A. Wall, K.-U. Bletzinger, E. Ramm, Models and Finite Elements for Thin-Walled Structures, in: E. Stein, R. d. Borst, T. J. R. Hughes (Eds.), Encyclopedia of computational mechanics, volume 2, Wiley, Chichester, 2004, pp. 59-137. 
[2] J. C. Simo, D. D. Fox, On a stress resultant geometrically exact shell model. Part I: Formulation and optimal parametrization, Comput. Meth. Appl. Mech. Engrg. 72 (1989) 267-304.

[3] J. C. Simo, D. D. Fox, M. S. Rifai, On a stress resultant geometrically exact shell model. Part II: The linear theory; Computational aspects, Comput. Meth. Appl. Mech. Engrg. 73 (1989) 53-92.

[4] J. C. Simo, D. D. Fox, M. S. Rifai, On a stress resultant geometrically exact shell model. Part III: Computational aspects of the nonlinear theory, Comput. Meth. Appl. Mech. Engrg. 79 (1990) 21-70.

[5] J. C. Simo, M. S. Rifai, D. D. Fox, On a stress resultant geometrically exact shell model. Part IV: Variable thickness shells with through-thethickness stretching, Comput. Meth. Appl. Mech. Engrg. 81 (1990) 91-126.

[6] W. Dornisch, S. Klinkel, B. Simeon, Isogeometric Reissner-Mindlin shell analysis with exactly calculated director vectors, Comput. Meth. Appl. Mech. Engrg. 253 (2013) 491-504.

[7] T. J. R. Hughes, J. A. Cottrell, Y. Bazilevs, Isogeometric analysis: CAD, finite elements, NURBS, exact geometry and mesh refinement, Comput. Meth. Appl. Mech. Engrg. 194 (2005) 4135-4195.

[8] J. Kiendl, K.-U. Bletzinger, J. Linhard, R. Wüchner, Isogeometric shell analysis with Kirchhoff-Love elements, Comput. Meth. Appl. Mech. Engrg. 198 (2009) 3902-3914.

[9] D. J. Benson, S. Hartmann, Y. Bazilevs, M.-C. Hsu, T. J. R. Hughes, Blended isogeometric shells, Comput. Meth. Appl. Mech. Engrg. 255 (2013) 133-146.

[10] D. J. Benson, Y. Bazilevs, M.-C. Hsu, T. J. R. Hughes, A large deformation, rotation-free, isogeometric shell, Comput. Meth. Appl. Mech. Engrg. 200 (2011) 1367-1378.

[11] D. J. Benson, Y. Bazilevs, M.-C. Hsu, T. J. R. Hughes, Isogeometric shell analysis: The Reissner-Mindlin shell, Comput. Meth. Appl. Mech. Engrg. 199 (2010) 276-289.

[12] W. Dornisch, S. Klinkel, The interpolation of the director vector for isogeometric Reissner-Mindlin shell analysis, in: J. Eberhardsteiner (Ed.), Proc. 6th European Congress on Computational Methods in Applied Sciences and Engineering, e-Book Full Papers, Vienna (Austria), pp. $4556-4565$.

[13] R. Echter, B. Oesterle, M. Bischoff, A hierarchic family of isogeometric shell finite elements, Comput. Meth. Appl. Mech. Engrg. 254 (2013) $170-180$.

[14] R. Bouclier, T. Elguedj, A. Combescure, Efficient isogeometric NURBS-based solid-shell elements: Mixed formulation and B-method, Comput. Meth. Appl. Mech. Engrg. 267 (2013) 86-110.

[15] N. Nguyen-Thanh, J. Kiendl, H. Nguyen-Xuan, R. Wüchner, K.-U. Bletzinger, Y. Bazilevs, T. Rabczuk, Rotation free isogeometric thin shell analysis using PHT-splines, Comput. Meth. Appl. Mech. Engrg. 200 (2011) 3410-3424.

[16] Q. Long, P. B. Bornemann, F. Cirak, Shear-flexible subdivision shells, Int. J. Numer. Meth. Engng 90 (2012) $1549-1577$.

[17] J. Kiendl, Y. Bazilevs, M.-C. Hsu, R. Wüchner, K.-U. Bletzinger, The bending strip method for isogeometric analysis of Kirchhoff-Love shell structures comprised of multiple patches, Comput. Meth. Appl. Mech. Engrg. 199 (2010) 2403-2416.

[18] L. Piegl, W. Tiller, The NURBS book, Monographs in visual communications, Springer, Berlin, second edition, 1997.

[19] T. J. R. Hughes, The finite element method: Linear static and dynamic finite element analysis, Dover Publ., Mineola and NY, reprint. edition, 2000.

[20] G. Golub, Numerical Methods for Solving Linear Least Squares Problems, Numer. Math. 7 (1965) 206-216.

[21] P. Betsch, A. Menzel, E. Stein, On the parametrization of finite rotations in computational mechanics, Comput. Meth. Appl. Mech. Engrg. 155 (1998) 273-305.

[22] A. Ibrahimbegovic, On the choice of finite rotation parameters, Comput. Meth. Appl. Mech. Engrg. 149 (1997) 49-71.

[23] A. Ibrahimbegovic, F. Frey, I. Kožar, Computational aspects of vector-like parametrization of three-dimensional finite rotations, Int. J. Numer. Meth. Engng. 38 (1995) 3653-3673.

[24] A. Ibrahimbegovic, R. L. Taylor, On the role of frame-invariance in structural mechanics models at finite rotations, Comput. Meth. Appl. Mech. Engrg. 191 (2002) 5159-5176.

[25] F. Gruttmann, R. Sauer, W. Wagner, Theory and numerics of three-dimensional beams with elastoplastic material behaviour, Int. J. Numer. Meth. Engng. 48 (2000) 1675-1702.

[26] W. Wagner, F. Gruttmann, A robust nonlinear mixed hybrid quadrilateral shell element, Int. J. Numer. Meth. Engng. 64 (2005) $635-666$.

[27] R. Schmidt, J. Kiendl, K.-U. Bletzinger, R. Wüchner, Realization of an integrated structural design process: analysis-suitable geometric modelling and isogeometric analysis, Comput. Visual Sci. 13 (7) (2010) 315-330.

[28] T. J. R. Hughes, A. Reali, G. Sangalli, Efficient quadrature for NURBS-based isogeometric analysis: Computational Geometry and Analysis, Comput. Meth. Appl. Mech. Engrg. 199 (2010) 301-313.

[29] S. Klinkel, F. Gruttmann, W. Wagner, A mixed shell formulation accounting for thickness strains and finite strain $3 \mathrm{~d}$ material models, Int. J. Numer. Meth. Engng. 74 (2008) 945-970.

[30] A. Apostolatos, R. Schmidt, R. Wüchner, K.-U. Bletzinger, A Nitsche-type formulation and comparison of the most common domain decomposition methods in isogeometric analysis, Int. J. Numer. Meth. Engng (2013) doi: 10.1002/nme.4568.

[31] R. Schmidt, R. Wüchner, K.-U. Bletzinger, Isogeometric analysis of trimmed NURBS geometries, Comput. Meth. Appl. Mech. Engrg. 241-244 (2012) 93-111. 